OPEN ACCESS

Edited by:

Philip P. Foster,

Baylor College of Medicine,

United States

Reviewed by:

Talitha Best,

Central Queensland University,

Australia

Claudia Voelcker-Rehage,

University of Münster, Germany

${ }^{*}$ Correspondence: Natáli Valim Oliver Bento-Torres natalivalim@ufpa.br

Received: 30 July 2020 Accepted: 20 January 2021

Published: 16 February 2021

Citation:

Jardim NY, Bento-Torres NVO,

Costa VO, Carvalho JPR,

Pontes HTS, Tomás AM,

Sosthenes MCK, Erickson Kl,

Bento-Torres J and Diniz CWP (2021)

Dual-Task Exercise to Improve

Cognition and Functional Capacity

of Healthy Older Adults.

Front. Aging Neurosci. 13:589299.

doi: 10.3389/fnagi.2021.589299

\section{Dual-Task Exercise to Improve Cognition and Functional Capacity of Healthy Older Adults}

\author{
Naina Yuki Vieira Jardim", Natáli Valim Oliver Bento-Torres ${ }^{1,2 *}$, Victor Oliveira Costa \\ Josilayne Patricia Ramos Carvalho ${ }^{1,2}$, Helen Tatiane Santos Pontes', \\ Alessandra Mendonça Tomás ${ }^{1}$, Marcia Consentino Kronka Sosthenes ${ }^{1}$, Kirk I. Erickson ${ }^{3}$, \\ João Bento-Torres ${ }^{1,2}$ and Cristovam Wanderley Picanço Diniz ${ }^{1}$ \\ ${ }^{1}$ Neurodegeneration and Infection Research Laboratory, Institute of Biological Science/João de Barros Barreto University \\ Hospital, Federal University of Pará, Belém, Brazil, ${ }^{2}$ Physical Therapy and Occupational Therapy Program, Federal University \\ of Pará, Belém, Brazil, ${ }^{3}$ Department of Psychology, University of Pittsburgh, Pittsburgh, PA, United States
}

Background: It has been suggested that physical inactivity and lack of stimulating cognitive activity are the two most significant modifiable risk factors to impair cognitive function. Although many studies that investigated the cognitive effects of physical exercise and cognitive stimuli in dual-task conditions showed improved cognitive performance, others have not confirmed these findings. The main aim of the present work is to analyze the effects of a dual-task multimodal physical exercise training, at moderate intensity, and cognitive stimulation on cognitive and physical function in healthy older adults.

Methods: This clinical trial was registered on the Brazilian Registry of Clinical Trials (RBR-9zrx3d). Here we tested the effects of a dual-task multimodal physical exercise training, at moderate intensity, on cognitive and physical function and quality of life in community dwelling older adults. The training protocol included 24 group sessions, 2/week, per 75 min. Cognition was assessed using CANTAB automated neuropsychological tests and Functional Capacity to Exercise tests. Performance was compared from baseline to post intervention and to a non-exercise control group using Mixed Linear Model for repeated measures.

Results: Control (CG) and dual-task (DTEx) groups progressed differentially over time on performance of episodic memory, sustained visual attention, functional mobility, cardiorespiratory fitness, lower limbs strength resistance, agility, quality of life and dualtask performance with significant improved DTEx performance. Control group did not show any significant changes on these tests except for showing a reduction in dual-task performance.

Conclusion: We suggest that the dual-task combination of multisensory cognitive stimulation and multimodal moderate physical exercise training, twice a week, may be 
adopted as an effective program to reduce progression of age-related cognitive decline and improve physical fitness and quality of life on healthy older adults.

Clinical Trial Registration: Brazilian Registry of Clinical Trials: https://ensaiosclinicos. gov.br/rg/RBR-9zrx3d -UTN code: U1111-1233-6349.

Keywords: cognitive dysfunction, rehabilitation, physical therapy modalities, aged, neuropsychological tests, healthy aging, dual-task exercise, physical fitness

\section{INTRODUCTION}

As we celebrate greater longevity, we are witnessing a change in the nosological profile that now includes higher incidence rates of neurodegenerative diseases associated with aging, including cognitive decline and dementia, of which the prevalence doubles every 6 years from the age of 65 (Cheng, 2016). Dementia implies high costs for health systems and great negative impact on quality of life for patients, as well as family members and caregivers (Prince et al., 2015). Further aggravating this scenario is that during the course of the last few generations there is greater availability of information technologies and mobile telecommunications with less expensive internet access, which has expanded the underlying trend of sedentary behavior (Owen et al., 2020). The increasing time spent sitting or lying down, sitting in automobiles or public transportation, combined with increased life expectancy, contributes to the increased risk and prevalence of type 2 diabetes (Garneau and Aguer, 2019), cardiovascular disease (Morris et al., 1953; Green et al., 2017), cancer (Ninot et al., 2020), musculoskeletal disability (Rezuş et al., 2020), as well as a broad range of other adverse health outcomes related to a sedentary lifestyle behavior (Owen et al., 2020) including the long term risk of dementia (Magnon et al., 2018; Ekblom et al., 2019; Coelho et al., 2020) and other age-associated neurodegenerative diseases (Kivimäki et al., 2019).

It is estimated that by 2050 the number of people living with dementia worldwide will increase from 47 to 132 million (Prince et al., 2015; Irwin et al., 2018). In line with this prediction, the global number of people living with dementia more than doubled from 1990 to 2016, mainly due to increases in population aging and growth (Nichols et al., 2019). Thus, healthy aging to prevent neurodegenerative diseases has become increasingly important for public health policy makers, and until breakthroughs are made in prevention or curative treatments, dementia will constitute an increasing challenge to health care systems worldwide (Nichols et al., 2019). Given the multifactorial etiology of dementia, simultaneous interventions in multiple domains might be critical for minimizing effects of various risk factors (Kivipelto et al., 2018) and for prevention and treatment of age-related cognitive decline.

Exercise programs are viable interventions to improve cognition and reduce cognitive decline (Stillman et al., 2020). It is an efficient, safe, and low-cost non-pharmacological strategy broadly investigated: aerobic and strength training or the combination of both, show positive effects on brain structure and function, behavior, and cognition (Erickson et al., 2019). Recommendations suggest multimodal training (Chodzko-Zajko et al., 2009; Gregory et al., 2013; Northey et al., 2018; Wollesen et al., 2020). This recommendation is based on the different types of training contributions to cognition (Liu-Ambrose and Donaldson, 2009; Forte et al., 2013) and physical function (Cadore and Izquierdo, 2013). It is suggested that physical training should be heart rate monitored and performed at moderate intensity (Lauenroth et al., 2016; Stojan and Voelcker-Rehage, 2019).

The differential efficiency of dual-task intervention in comparison to single stimulation is unclear (Bruderer-Hofstetter et al., 2018; Guo et al., 2020), but in healthy older adults the simultaneous conduction of a mental and a physical task (dual-task) seems to be particularly efficient to increase cognitive function (Eggenberger et al., 2015; Falbo et al., 2016; Lauenroth et al., 2016; Tait et al., 2017; Gheysen et al., 2018; Herold et al., 2018), in particular if mentally challenging activities are simultaneously performed with multimodal physical exercises (Gheysen et al., 2018; Li et al., 2018; Northey et al., 2018). However, because of the wide variation between protocols, the lack of detailed methodological description (e.g., baseline levels of physical fitness, and training intensity) and small sample sizes in some studies (Stojan and Voelcker-Rehage, 2019; Guo et al., 2020), there is still a need for more research for an optimal protocol and information on detraining effects (Gheysen et al., 2018).

Accurate and precise tools for assessing cognition contribute to more robust inferences about the influence of interventions on cognition. In addition, it is necessary to assess cognitive functions that are altered across lifespan, such as episodic memory (Korkki et al., 2020), working memory (Cansino et al., 2020) and attention (McDonough et al., 2019). To go for it, we used automated computerized neuropsychological tests with great specificity and sensitivity (De Jager et al., 2002; Wild et al., 2008; Cabral Soares et al., 2015). Compared with traditional standard methods, automated computer tests minimize floor and ceiling effects, standardize the format of application, and measure the speed and accuracy of responses with greater sensitivity and specificity (Wild et al., 2008).

Because cognitive effects of exercise and cognitive stimulation in dual-task paradigms are ambiguous (Ansai et al., 2017; Tait et al., 2017; Guo et al., 2020; Wollesen et al., 2020) there is a need for more sensitive cognitive testing batteries rather than using screening tests as outcome measures to assess the influence of dual-task interventions (Wollesen et al., 2020). Thus, with a highly sensitive evaluation battery, we have gathered quantitative 
and qualitative characteristics based on the most recent recommendations to investigate whether a dual-task protocol following these guidelines is effective. We hypothesized that a moderate-intensity multimodal exercise program performed simultaneously to a complex previously validated cognitive stimulation is an effective intervention program to improve verbal and visual episodic memory, sustained visual attention, physical function, and quality of life of healthy older adults. We expected to find that control (CG) and dual-task (DTEx) groups would progress differentially over time on performance of physical and cognitive tasks.

\section{MATERIALS AND METHODS}

\section{Overview}

This clinical trial was registered on the Brazilian Registry of Clinical Trials (RBR-9zrx3d). Participants were allocated not randomly to either the Dual-Task Exercise (DTEx) or Control (CG) groups. All participants were given the opportunity to choose to take part into DTEx or CG prior to baseline assessments, based on their own possibility to come to the intervention facility and stay committed with regular session attendance. The factors that determined the participants choice on groups included the home distance to intervention facility (the main reason in the most cases), incompatibility with the training session schedule and, for a small number of participants also reported difficulties in transportation to intervention site. The DTEx participated in a 24-session intervention protocol with physical exercise training and simultaneous cognitive stimulation (Dual-task). The Control (CG) group participants received educational materials on health-related topics. Both groups were instructed to maintain their daily routine. Both groups were evaluated before and after a 3-month intervention period. This study was approved by the Health Science Institute of Federal University of Pará Review Board (CAAE no. 03427318.3.0000.0018).

\section{Participants}

Participants were community dwelling, healthy older adults invited to participate by advertisements on social media, seniors' centers, health care units and the University surrounding community. To be eligible, participants were required to be $>59$ years old and cognitively healthy according to a Mini Mental State Examination (MMSE; 0-30 points) cutoff score adjusted to educational levels for the Brazilian population as follows: illiterate, $13 ; 1-7$ years of schooling, $18 ; \geq 8$ years of schooling, 26 (Bertolucci et al., 1994). They also had to report no history of traumatic brain injury, stroke, or depression and be physically inactive (no regular practice of physical exercise) for at least 6 months prior to the assessments. In addition, visual acuity of $20 / 30$ or better (Snellen test) was adopted as inclusion criteria to reduce possible biases in cognitive performance associated with visual impairments. All participants provided written informed consent before data collection. We also required written statements from the primary care physician indicating that the participant was safe to participate in an exercise program.

To determine the appropriate sample size needed to test our aims, we used G Power 3.1 software $^{1}$ using a priori power analysis. Considering that previous meta-analyses have reported small-to-moderate sized effects of physical exercise on cognitive performance in older adults (Colcombe and Kramer, 2003; Zhu et al., 2016), we estimated a conservative effect size of $f=0.20$. We based the calculation on a statistical power of $85 \%$, a two-sided hypothesis test, an alpha level of 0.05 and an analysis of variance model, within-between interaction. Sample size calculation resulted in at least 60 participants, 30 in each group. Estimating 20\% dropout, the final size was calculated at 36 participants per group.

Participants completed all the cognitive, functional exercise capacity tests and quality of life assessments in a single day, before and after the intervention period. On average, tests were completed within $90 \mathrm{~min}$ and a brief break was offered between tests. Initially, 312 older adults were invited to participate, of which 221 were excluded based on eligibility criteria, not consenting to the procedures, or absence to the scheduled assessment. Thus, 91 participants were divided into DTEx $(n=55)$ and CG $(n=36)$. Over the 3 -month intervention period, 14 participants from the intervention group and 5 from the CG dropped-out to participating in fewer than $75 \%$ of the sessions, health problems unrelated to the intervention, withdrawal from the study, or other reasons. The final analysis included 41 older adults in DTEx and 31 in CG (Figure 1). Data were collected at the Federal University of Pará Facilities, in the same testing room with control for noise, luminosity and temperature.

\section{Cognitive Assessment}

The MMSE was used for eligibility purposes as described above (Bertolucci et al., 1994, 2001). The CERAD Word List Memory was used to assess episodic verbal memory (immediate memory and word list delayed recall) and recognition memory. The tests were applied according to Bertolucci et al. (1998).

Cambridge Neuropsychological Test Automated Battery (CANTAB) is an automated test battery used for cognitive assessment in the present study. CANTAB is less influenced by the administrator due its non-verbal stimuli and responses required. In addition, they are visually attractive and have an immediate feedback tool similar to games, which maintains motivation. It is also possible to estimate response times with millisecond precision, avoiding the compression of scales (Sahakian and Owen, 1992). In addition, studies suggest that automated tests are a more sensitive tool for identification of subtle variations in cognitive performance (De Jager et al., 2002; Wild et al., 2008). The Motor Screening Test (MOT) was performed to assess the ability to understand and complete tasks using the touchscreen technology.

Episodic memory was assessed by PAL. During the PAL test, 8 boxes were uncovered, one by one, in a randomized order, to reveal either an empty space or a figure. Then each figure was individually displayed on the center of the screen

\footnotetext{
${ }^{1}$ http://www.gpower.hhu.de
} 


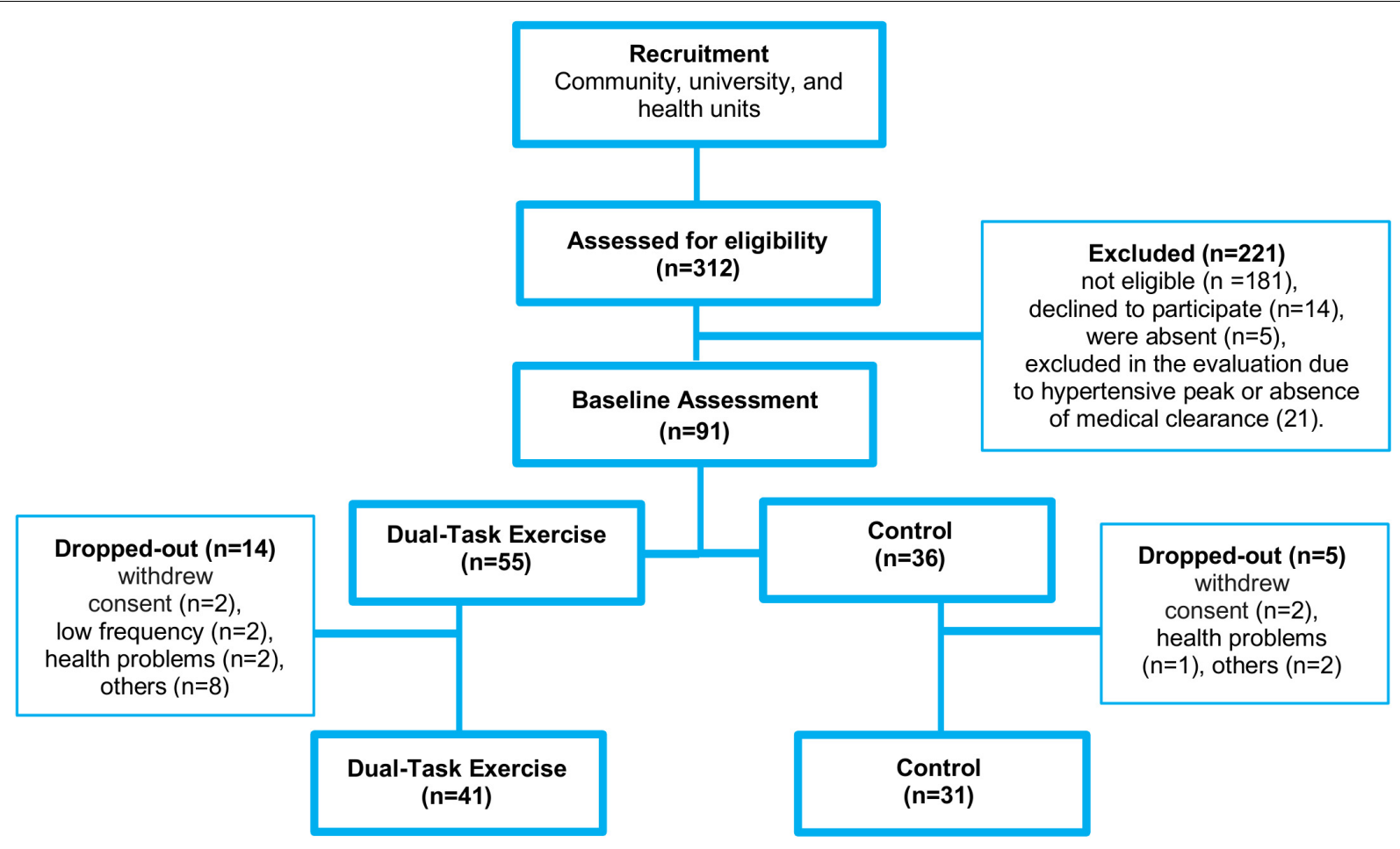

FIGURE 1 | Flow diagram of recruitment, allocation, intervention, and final sample included in data analysis.

and the participant was instructed to indicate the location in which the figure was originally exposed. In case of error, the figures were presented again for up to 10 attempts. Initially, 2 figures were presented, and, to the extent of success, the number of figures increased to 3,6 and then up to 8 . PAL test administration time is around $7-10 \mathrm{~min}$, but largely depends on the participant's performance and number of repeat presentations required. There is no limited response time and because of that no predetermined inter-stimulus interval. PAL output variables included: PAL Stages completed (PAL SC), which corresponds to the number of completed steps, and is a key result of test success, a higher score is better; PAL Number of patterns succeeded (PAL NPS), which corresponds to the number of pairs successfully completed in the last stage that the participant performed, a higher score is better; and PAL Total Trials Adjusted (PAL TTA), which represents the total number of figure presentations required for the participant to answer correctly (maximum 10 presentations), a lower score is better (Cambridge Cognition, 2016).

RVP assesses the ability to sustain visual attention. In a central rectangle, several subsequent and random numbers were displayed, at the rate of 100 digits per minute. In the training stage, the volunteer was instructed to press a pad after a target number sequence (3-5-7). In the test stage, the participant was instructed to press the pad after any of three target sequences (3-5-7, 2-4-6, or 4-6-8). The administration time is around 7 to $10 \mathrm{~min}$. Output measurements were RVP Mean latency, which details the average response time in milliseconds. This measure only includes correct responses within an 1,800 ms response window. RVP latency is a good indicator of sustained attention (Cognition, 2014).

\section{Physical Activity Assessment}

Self-reported physical activity data were collected using the International Physical Activity Questionnaire (IPAQ, version 8, long form) in an interview (Benedetti et al., 2004). The IPAQ consists of 27 questions that quantifies physical activity, in a typical week, considering only activities performed for at least $10 \mathrm{~min}$. Participants were asked to report the time spent in physical activity performed across work, domestic activities, exercise/leisure time, and transport at each of 3 intensities: walking, moderate, and vigorous. Scoring procedures followed the Guideline for data processing and Analysis of the IPAQ 2 . Results are expressed in Metabolic Equivalents-minutes per week (MET min/week).

\section{Functional Exercise Capacity Assessment}

Functional exercise capacity assessments included measures of Functional Mobility (Timed Up and Go Test-TUG), an indirect measure of cardiorespiratory fitness (Six-Minute Walk Test6MWT), lower limb strength resistance (30-seconds Chair Stand Test-30 CST). Briefly: TUG measures the total time (in seconds) the participant rises from an armless chair, walks $3 \mathrm{~m}$, and returns to sit position (Podsiadlo and Richardson, 1991); 6MWT assesses the distance walked on a flat, hard surface, in a period of

\footnotetext{
${ }^{2}$ https://sites.google.com/site/theipaq/scoring-protocol
} 
$6 \mathrm{~min}$. The participants were instructed to walk at their own pace. Speed changes were allowed. Standardized incentive phrases were used throughout the test. The total distance walked (in meters) during 6 min was used for performance comparison between groups (American Thoracic Society, 2002). On the 30 CST test, the number of times the participant gets up from a sitting position on an armless chair is recorded for $30 \mathrm{~s}$. If necessary, ergonomic adjustments were made to ensure proper positioning (Jones et al., 1999).

Walking while talking test (Hall et al., 2011) was used to assess dual-task Agility by using walking speed during the task and verbal fluency, as a cognitive addition to the motor task. Agility was also measured by walking speed as a single task.

\section{Quality of Life Assessment}

Quality of life was assessed using the 36-Item Short Form Health Survey (SF-36). It is a self-reporting multidimensional instrument for routine monitoring and assessment of care outcomes in adults, encompassed in 8 scales or domains: functional capacity, physical aspects, pain, general health status, vitality, social aspects, emotional aspects and mental health, each domain assumes values from 0 (worst) to 100 (best) (Ciconelli et al., 1999).

\section{Physical Exercise in a Dual-Task Intervention Program}

The intervention program was composed of 24 intervention group sessions. Each group had a maximum of 15 participants and took place at community centers. Training sessions included warm-up (10 $\mathrm{min})$, aerobic exercise $(30 \mathrm{~min})$, resistance exercise (30 $\mathrm{min})$, and stretching ( $5 \mathrm{~min}$ ). In all sessions, participants used individual cardiofrequencimeter (Polar FT1 Heart Rate Sensor) to monitor the cardiovascular training zone and were encouraged to exercise at a moderate intensity $(60-70 \%$ of the maximum heart rate estimated using the Karvonen formula).

The warm up was performed by walking at different speeds associated with upper limbs movements. Aerobic training was performed in alternating sessions, consisting of walking exercises associated with a functional circuit, agility (zigzag between cones, carrying weights from one end to the other) and gait. Balance (walking on different surfaces, single leg support) and coordination exercises (throwing a ball into a basket, walking while passing a ball under the legs) were also performed. In the alternative session the participants practiced a dance choreography that would be resumed the following week. Regional musical styles that were pleasing to the participants were used. The cognitive training component, aerobic, balance and coordination tasks of the Dance training was met with the choreography learning and execution itself.

Resistance training prioritized multiarticular and global exercises such as squat and bench press. Periodization was defined according to American College of Sports Medicine recommendations, increasing the number of repetitions every 2 weeks starting with 3 series of 10 repetitions followed by 3 series of 15 repetitions and increased workload on
3 series of 10 repetitions. On session 12 changes to the training program were done and periodization progress repeated until session 24 (Garber et al., 2011). It is important to note that all exercise sessions were performed simultaneously with cognitive tasks. The full description of the DualTask intervention Program is described in Table 1. All sessions were run by two no blinded researchers with degrees as Physical Therapist and Bachelor's in Physical Education. Two Health/Sport Science graduate students helped during the sessions.

\section{Cognitive Stimulation}

The cognitive stimulation protocol associated with physical exercise included a multisensorial stimuli protocol based on a previous study published by our group (De Oliveira et al., 2014) (Table 1). To generate a multisensory stimulation, a complex program was developed including visual, auditory, and olfactory stimuli activities. The tasks mainly included functional responses to sensory stimuli, verbal and visual memory, motor learning, speech, attention, inhibition, and semantic and phonological fluency and were performed simultaneously to physical exercise training.

\section{Statistical Analysis}

The primary aim of this study was to investigate improvements in cognitive performance for the DTEx group in comparison to the CG. Secondary outcomes were functional exercise capacity and quality of life measures.

Prior to statistical analysis, outlier values $( \pm 2 \mathrm{SD}$ from the mean) were excluded. Age, education, and physical activity level (IPAQ) were compared using the Student $t$-test or MannWhitney based on the distribution. Differences between groups in relation to changes in cognitive, physical and quality of life parameters before and after the intervention were carried out using a Mixed Linear Model for repeated measures, in which time was considered as a within-subjects factor (pre and post) and group was treated as a between subjects factor (DTEx, CG). Bonferroni was used as a post hoc test. Effect size, as a measure of Intervention effect, were estimated. For this, the individual final pos-test score was subtracted from the group pre-test score average and divided by the standard deviation of the pre-test score (Sink et al., 2015). Thus, a $Z$ score was obtained for each outcome measure for each group. To compare DTEx and CG interventions effects ( $z$ score) an analysis of covariance ANCOVA was performed adjusting for age, education, and sex, using only the group (DTEx, CG) as the factor of analysis. For outcome measures (PAL TTA, RVP ML, and TUG) in which a negative effect was indicative of better test performance any negative effect sizes were multiplied by $(-1)$ to facilitate interpretation. Effect sizes were classified based on Z Score: 0.00-0.19-trivial; 0.20-0.49-small, 0.50-0.79moderate; $\geq 0.80$-large (Cohen, 1992). Analyses were performed using IBM SPSS Statistics version 20 (Armonk, Nova York: IBM Corporation). 
TABLE 1 | Dual-task intervention program protocol.

\begin{tabular}{|c|c|c|}
\hline Sessions & Stimulus & Cognitive tasks simultaneous to exercise \\
\hline \multirow[t]{2}{*}{1} & Speech & $\begin{array}{l}\text { The group was encouraged to speak out loudly the days of the week, months of the year, and } \\
\text { the alphabet in direct and reverse order. }\end{array}$ \\
\hline & Long-term memory & $\begin{array}{l}\text { Participants were asked to remember pre-selected words and sing songs with the vocabulary } \\
\text { included. }\end{array}$ \\
\hline \multirow[t]{2}{*}{2} & Short-term memory & $\begin{array}{l}\text { At functional physical exercise circuit beginning, researcher reads a word sequence (places, } \\
\text { animals, and objects), which were requested to be reproduced by the participant at the circuit } \\
\text { endpoint. }\end{array}$ \\
\hline & Mathematical thinking & Simple calculations (arithmetic). \\
\hline \multirow[t]{2}{*}{3} & Motor learning & Learning a new choreography \\
\hline & $\begin{array}{l}\text { Semantic and phonological } \\
\text { fluency }\end{array}$ & $\begin{array}{l}\text { The group was encouraged to remember and to speak out loud words from a certain } \\
\text { category or phoneme. }\end{array}$ \\
\hline \multirow[t]{2}{*}{4} & Mathematical thinking & Simple calculations (arithmetic). \\
\hline & Reasoning & $\begin{array}{l}\text { The participants were encouraged to deduce a hidden word on the board. Researchers } \\
\text { provided clues. }\end{array}$ \\
\hline \multirow[t]{2}{*}{5} & $\begin{array}{l}\text { Long-term memory and Motor } \\
\text { learning }\end{array}$ & Remember the previous choreography \\
\hline & Speech & Participants were asked to do storytelling and, collaboratively, create a new story. \\
\hline \multirow[t]{2}{*}{6} & $\begin{array}{l}\text { Attention, Decision-making and } \\
\text { Short-term memory }\end{array}$ & $\begin{array}{l}\text { Specific sound stimulus (one whistle, two whistles, and applause) were associated with a } \\
\text { specific sequence of motor tasks to be performed. }\end{array}$ \\
\hline & $\begin{array}{l}\text { Short-term memory and } \\
\text { Attention }\end{array}$ & $\begin{array}{l}\text { One of the participants started a sequence of grocery store shopping list saying: "I went to the } \\
\text { grocery and bought an..." (e.g., apple). The closest participant was asked to repeat the } \\
\text { previous statement and add a new item to the shopping list. The process was continuous and } \\
\text { items cumulative until everyone in the group had contributed. }\end{array}$ \\
\hline \multirow[t]{2}{*}{7} & Motor learning & Learning a new choreography \\
\hline & Reasoning & $\begin{array}{l}\text { The participants were encouraged to deduce a hidden word on the board. Randomly selected } \\
\text { participant provided clues. }\end{array}$ \\
\hline \multirow[t]{2}{*}{8} & $\begin{array}{l}\text { Attention, decision-making, and } \\
\text { inhibition }\end{array}$ & $\begin{array}{l}\text { At the sound of a whistle, participants should perform a sequence of pre-learned motor tasks. } \\
\text { At the sound of two whistles, they should perform the movement illustrated among other } \\
\text { equal distractors from a projected image. }\end{array}$ \\
\hline & Inhibition and Processing speed & Stroop Test \\
\hline \multirow[t]{2}{*}{9} & $\begin{array}{l}\text { Long-term memory and motor } \\
\text { learning }\end{array}$ & Remember the previous choreography \\
\hline & $\begin{array}{l}\text { Long-term memory and } \\
\text { autobiographical }\end{array}$ & $\begin{array}{l}\text { Old singers' photos and songs were placed, and the group was asked to identify the singer, } \\
\text { named the song, and asked to share personal experiences evoked by the music. }\end{array}$ \\
\hline \multirow[t]{2}{*}{10} & $\begin{array}{l}\text { Attention, decision-making, and } \\
\text { short-term memory }\end{array}$ & $\begin{array}{l}\text { Specific sound stimulus (one whistle, two whistles, and applause) were associated with a } \\
\text { specific sequence of motor tasks to be performed. }\end{array}$ \\
\hline & Speech & $\begin{array}{l}\text { The group was encouraged to speak out loudly the days of the week, months of the year and } \\
\text { the alphabet in direct and reverse order. }\end{array}$ \\
\hline \multirow[t]{2}{*}{11} & Motor learning & Learning a new choreography \\
\hline & Mathematical reasoning & Simple mathematical problems \\
\hline \multirow[t]{2}{*}{12} & Short-term memory & $\begin{array}{l}\text { At functional physical exercise circuit beginning, researcher reads a word sequence (places, } \\
\text { animals, and objects), which were requested to be reproduced by the participant at the circuit } \\
\text { endpoint. }\end{array}$ \\
\hline & Speech & Participants were asked to do storytelling and, collaboratively, create a new story. \\
\hline \multirow[t]{2}{*}{13} & $\begin{array}{l}\text { Long-term memory and motor } \\
\text { learning }\end{array}$ & Remember the previous choreography \\
\hline & Inhibition and processing speed & Stroop Test \\
\hline \multirow[t]{2}{*}{14} & $\begin{array}{l}\text { Long-term memory and } \\
\text { short-term memory }\end{array}$ & $\begin{array}{l}\text { At functional physical exercise circuit beginning, specific sequence of olfactory stimuli were } \\
\text { presented. Later, the identification and the sequence of the odors presented were requested. }\end{array}$ \\
\hline & $\begin{array}{l}\text { Short-term memory and } \\
\text { attention }\end{array}$ & $\begin{array}{l}\text { One of the participants started a sequence of grocery store shopping list saying: "I went to the } \\
\text { grocery and bought an ..." (e.g., apple). The closest participant was asked to repeat the } \\
\text { previous statement and add a new item to the shopping list. The process was continuous and } \\
\text { items cumulative until everyone in the group had contributed. }\end{array}$ \\
\hline \multirow[t]{2}{*}{15} & Motor learning & Learning a new choreography \\
\hline & $\begin{array}{l}\text { Attention and short-term } \\
\text { memory }\end{array}$ & The researcher reads news and proceeds a group discussion about the information read. \\
\hline
\end{tabular}

\section{Exercise}

Functional Circuit + Walking

Resistance training

Functional

Circuit + Walking

Resistance training

Dance

Resistance training

Functional

Circuit + Walking

Resistance training

Dance

Resistance training

Functional

Circuit + Walking

Resistance training

Dance

Resistance training

Functional

Circuit + Walking

Resistance training

Dance

Resistance training

Functional

Circuit + Walking

Resistance training

Dance

Resistance training

Functional

Circuit + Walking

Resistance

Dance

Resistance training

Functional

Circuit + Walking

Resistance training

Dance

Resistance training 
TABLE 1 | Continued

\begin{tabular}{|c|c|c|c|}
\hline Sessions & Stimulus & Cognitive tasks simultaneous to exercise & Exercise \\
\hline \multirow[t]{2}{*}{16} & $\begin{array}{l}\text { Attention and long-term } \\
\text { memory }\end{array}$ & $\begin{array}{l}\text { A song was played during the exercise, and then suddenly interrupted. Participants were } \\
\text { encouraged to continue to sing, completing the song. }\end{array}$ & $\begin{array}{l}\text { Functional } \\
\text { Circuit }+ \text { Walking }\end{array}$ \\
\hline & $\begin{array}{l}\text { Inhibition and processing } \\
\text { speed }\end{array}$ & Stroop Test & Resistance training \\
\hline \multirow[t]{2}{*}{17} & $\begin{array}{l}\text { Long-term memory and } \\
\text { motor learning }\end{array}$ & Remember the previous choreography & Dance \\
\hline & $\begin{array}{l}\text { Attention and short-term } \\
\text { memory }\end{array}$ & $\begin{array}{l}\text { A music was played and after that the group was asked to identify among projected images, } \\
\text { the objects mentioned by the music. Confounders images were used. }\end{array}$ & Resistance training \\
\hline \multirow[t]{2}{*}{18} & $\begin{array}{l}\text { Long-term memory and } \\
\text { short-term memory }\end{array}$ & $\begin{array}{l}\text { Commemorative dates were said at the beginning of the functional circuit and requested to be } \\
\text { mentioned at the end. }\end{array}$ & $\begin{array}{l}\text { Functional } \\
\text { Circuit }+ \text { Walking }\end{array}$ \\
\hline & Emotional prosody & Identification of emotions on the images displayed. & Resistance \\
\hline \multirow[t]{2}{*}{19} & Motor learning & Learning a new choreography & Dance \\
\hline & Attention and reasoning & $\begin{array}{l}\text { One word was given for a random participant, who had to describe characteristics of his word } \\
\text { so the others could try to guess it. }\end{array}$ & Resistance training \\
\hline \multirow[t]{2}{*}{20} & $\begin{array}{l}\text { Long-term memory and } \\
\text { short-term memory }\end{array}$ & $\begin{array}{l}\text { At functional physical exercise circuit beginning, Specific sequence of olfactory stimuli were } \\
\text { presented. Later, the identification and the sequence of the odors presented were requested. }\end{array}$ & $\begin{array}{l}\text { Functional } \\
\text { Circuit }+ \text { Walking }\end{array}$ \\
\hline & Short-term memory & Visual memory game with increased degree of difficulty. & Resistance \\
\hline \multirow[t]{2}{*}{21} & $\begin{array}{l}\text { Long-term memory and } \\
\text { motor learning }\end{array}$ & Remember the previous choreography & Dance \\
\hline & $\begin{array}{l}\text { Long-term memory and } \\
\text { autobiographical }\end{array}$ & $\begin{array}{l}\text { Old singers' photos and songs were placed, and the group was asked to identify the singer, } \\
\text { named the song, and asked to share personal experiences evoked by the music. }\end{array}$ & Resistance training \\
\hline \multirow[t]{2}{*}{22} & $\begin{array}{l}\text { Sustained attention and } \\
\text { decision-making }\end{array}$ & At a specific sound, the group should change the motor task. There were confounding sounds. & $\begin{array}{l}\text { Functional } \\
\text { Circuit }+ \text { Walking }\end{array}$ \\
\hline & $\begin{array}{l}\text { Long-term memory and } \\
\text { reasoning }\end{array}$ & Questions about general knowledge issues. & Resistance training \\
\hline \multirow[t]{2}{*}{23} & Motor learning & Learning a new choreography & Dance \\
\hline & Emotional prosody & Identification of emotions on the images displayed. & Resistance \\
\hline \multirow[t]{2}{*}{24} & $\begin{array}{l}\text { Long-term memory and } \\
\text { Motor learning }\end{array}$ & Remember the previous choreography & Dance \\
\hline & $\begin{array}{l}\text { Semantic and phonological } \\
\text { fluency }\end{array}$ & $\begin{array}{l}\text { The group was encouraged to remember and to speak out loud words from a certain category } \\
\text { or phoneme. }\end{array}$ & Resistance training \\
\hline
\end{tabular}

\section{RESULTS}

Seventy-two participants were included in the final analysis: 41 (36 female) in the DTEx and 31 (25 female) in the CG. Groups were matched for age $(\mathrm{DTEx}=67.39 \pm 0.90, \mathrm{CG}=67.87 \pm 0.99$, $p=0.56$ years $)$, education $($ DTEx $=8.64 \pm 0.63$, $\mathrm{CG}=8.64 \pm 0.79, p=0.99$ years) and physical activity level $($ DTEx $=626.18 \pm 107.90, C G=1304.85 \pm 344.07$, $p=0.27$ SUM-IPAQ- MET-min/week). No adverse effects or complications related to the exercise intervention occurred.

\section{Cognitive Performance Results}

Mixed Linear models were applied to each test score. Results revealed that the intervention positively influenced episodic memory (PAL test) and sustained visual attention (RVP test).

Main effects of Time were detected $\left[F_{(1,67)}=5.847, p=0.018\right]$ and a significant Group $x$ Time interaction was detected for PAL SC $\left[F_{(1,67)}=4.038, p=0.049\right]$, demonstrating that PAL was differentially affected in the CG and DTEX over time. Post hoc tests indicated that only the DTEx group showed significant improvements in episodic memory by increasing the number of stages completed in the test $(p=0.001)$.
In contrast, there was no change for the CG $(p=0.790)$. Main effects of Time were also observed for episodic memory assessed by a decrease in Total Trials Adjusted [PAL TTA: $\left.F_{(1.67)}=17.079, p<0.001\right]$ and an increase in the number of patterns succeeded [PAL NPS: $F_{(1.66)}=6.647, p=0.012$ ], with significant DTEx improvements for both PAL TTA $(p<0.001)$ and PAL NPS $(p=0.001)$ while there were no changes for the CG (PAL TTA: $p=0.150$; PAL NPS: $p=0.652$ ) (Table 2).

Taken together, these results indicate that trained participants improved cognitive performance demonstrated by the increased number of steps completed in the test (PAL SC), hit a greater number of pairs in the last step performed (PAL NPS) and decreased the required number of figures presentations (PAL TTA).

Main effects of time were also detected for sustained visual attention (RVP test) $\left[F_{(1.66)}=7.609, p=0.008\right]$ with no Group $\times$ Time interaction $\left[F_{(1.66)}=1.590, p=0.212\right]$. Post hoc tests indicated significant improvements for the DTEx group as reflected by a decrease in the mean latency to correctly answer the test's target sequence $(p=0.004)$, while there was no differences for the CG $(p=0.314)$ (Table 2). 
TABLE 2 | Cognitive performance scores on pre- and post-tests.

\begin{tabular}{|c|c|c|c|c|c|c|c|c|c|c|}
\hline & \multirow[t]{2}{*}{ Group } & \multirow[t]{2}{*}{ Pre-test } & \multirow[t]{2}{*}{ Pos-test } & \multirow[t]{2}{*}{ IC (95\%) } & \multicolumn{2}{|c|}{ Interaction } & \multicolumn{2}{|c|}{ Time } & \multicolumn{2}{|c|}{ Group } \\
\hline & & & & & $F$-value & Partial $\eta_{p}^{2}$ & $F$-value & Partial $\eta_{p}^{2}$ & $F$-value & Partial $\eta_{p}^{2}$ \\
\hline Episodic memory & CG & $4.07 \pm 0.14$ & $4.10 \pm 0.11$ & $-0.292-0.223$ & $4.038^{*}$ & 0,057 & $5.847^{\star}$ & 0,080 & 1.170 & 0,017 \\
\hline (PAL SC-score) & DTEx & $4.05 \pm 0.12$ & $4.42 \pm 0.10^{\# \star \star \star}$ & $-0.594--0.156$ & & & & & & \\
\hline Episodic memory & $\mathrm{CG}$ & $5.90 \pm 0.32$ & $6.03 \pm 0.25$ & $-0.745-0.469$ & 3.577 & 0,051 & $6.647^{\star \star}$ & 0,091 & 2.506 & 0,037 \\
\hline (PAL NPS-score) & DTEx & $6.03 \pm 0,28$ & $6.92 \pm 0.21^{\# \# \star \star * *}$ & $-1.421--0.374$ & & & & & & \\
\hline Episodic memory & $\mathrm{CG}$ & $19.83 \pm 0.97$ & $18.52 \pm 0.88$ & $-0.486-3.107$ & 3.670 & 0,052 & $17.079^{\star \star \star}$ & 0,203 & 0.473 & 0,007 \\
\hline (PAL TTA-score) & DTEx & $20.22 \pm 0.83$ & $16.65 \pm 0.75^{\star \star \star}$ & $2.045-5.105$ & & & & & & \\
\hline Sustained attention & $\mathrm{CG}$ & $713.54 \pm 39.99$ & $675.29 \pm 30.63$ & $-36.998-113.498$ & 1.590 & 0,024 & $7.609^{\star \star}$ & 0,103 & 3.476 & 0,050 \\
\hline (RVP ML-score) & DTEx & $669.36 \pm 36.60$ & $566.68 \pm 28.03^{\# \star \star}$ & $33.808-171.562$ & & & & & & \\
\hline Immediate memory & CG & $16.32 \pm 0.63$ & $17.84 \pm 0.70^{\star \star}$ & $-2.552--0.480$ & 3.246 & 0,044 & $38.534^{\star \star \star}$ & 0,355 & 1.910 & 0,027 \\
\hline (Word list - CERAD) & DTEx & $16.83 \pm 0.55$ & $19.58 \pm 0.61^{\star \star \star}$ & $-3.657--1.855$ & & & & & & \\
\hline Evocation memory & $\mathrm{CG}$ & $4.83 \pm 0.36$ & $5.40 \pm 0.38$ & $-1.173-0.040$ & 1.453 & 0,021 & $16.309^{\star \star \star}$ & 0,191 & 3.778 & 0,052 \\
\hline (Word list - CERAD) & DTEx & $5.46 \pm 0.31$ & $6.51 \pm 0.33^{\# \star \star \star}$ & $-1.568--0.530$ & & & & & & \\
\hline Recognition memory & CG & $8.64 \pm 0.24$ & $8.57 \pm 0.21$ & $-0.455-0.598$ & $4.258^{\star}$ & 0,063 & 2.736 & 0,042 & $6.888^{\star \star}$ & 0,099 \\
\hline (Word list-CERAD) & DTEX & $8.92 \pm 0.21$ & $9.57 \pm 0.18^{\# \# \# \star \star}$ & $-1.106--0.191$ & & & & & & \\
\hline
\end{tabular}

Mixed linear model was used. Values are presented with mean $\pm S E(I C=$ pre-test vs. pos-test).

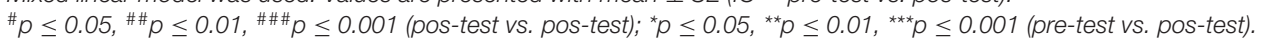

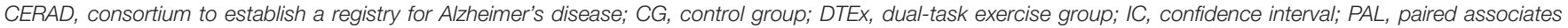

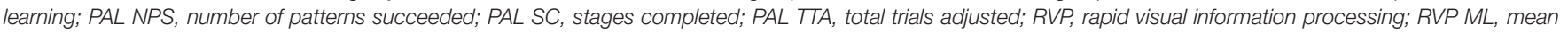
latency; SE, standard error.

TABLE 3 | Functional Exercise Capacity performance scores on pre- and post-tests.

\begin{tabular}{|c|c|c|c|c|c|c|c|c|c|c|}
\hline & \multirow[t]{2}{*}{ Group } & \multirow[t]{2}{*}{ Pre-test } & \multirow[t]{2}{*}{ Pos-test } & \multirow[t]{2}{*}{ IC (95\%) } & \multicolumn{2}{|c|}{ Interaction } & \multicolumn{2}{|c|}{ Time } & \multicolumn{2}{|c|}{ Group } \\
\hline & & & & & $F$-value & $\begin{array}{c}\text { Partial } \\
\eta_{p}^{2}\end{array}$ & $F$-value & $\begin{array}{c}\text { Partial } \\
\eta_{p}^{2}\end{array}$ & $F$-value & $\begin{array}{c}\text { Partial } \\
\eta_{p}^{2}\end{array}$ \\
\hline Cardiorespiratory & CG & $406.96 \pm 12.08$ & $387.46 \pm 14.27$ & $-2.798-41.798$ & $14.265^{\star \star \star}$ & 0,195 & 1.105 & 0,018 & $12.273^{\star \star \star}$ & 0,172 \\
\hline fitness (6MWT-meter) & DTEX & $433.86 \pm 9.73$ & $468.40 \pm 11.50^{\# \# \# \star \star \star \star}$ & $-52.499--16.582$ & & & & & & \\
\hline Functional mobility & CG & $8.69 \pm 0.30$ & $8.73 \pm 0.27$ & $-0.528-0.451$ & 8.970 ** & 0,128 & $7.587^{* *}$ & 0,111 & $13.968^{\star \star \star}$ & 0,186 \\
\hline (TUG-second) & DTEX & $7.919 \pm 0.252$ & $7.00 \pm 0.224^{\# \# \# * \star \star \star}$ & $0.508-1.330$ & & & & & & \\
\hline $\begin{array}{l}\text { Lower limbs strength } \\
\text { resistance }\end{array}$ & CG & $9.07 \pm 0.42$ & $9.70 \pm 0.40$ & $-1.414-0.155$ & $16.570^{\star \star \star}$ & 0,211 & $42.399^{\star \star \star}$ & 0,406 & $25.296^{\star \star \star}$ & 0,290 \\
\hline (30 CST-repetition) & DTEX & $10.40 \pm 0.36^{+}$ & $13.13 \pm 0.34^{\# \# \# \star \star \star \star}$ & $-3.400--2.060$ & & & & & & \\
\hline Agility & CG & $1.22 \pm 0.04$ & $1.24 \pm 0.04$ & $-0.117-0.069$ & $6.323^{\star \star}$ & 0,097 & $10.937^{\star \star}$ & 0,156 & $14.452^{\star \star \star}$ & 0,197 \\
\hline$(\mathrm{m} / \mathrm{s})$ & DTEX & $1.30 \pm 0.03$ & $1.48 \pm 0.03^{\# \# \# \star \star \star \star}$ & $-0.249--0.099$ & & & & & & \\
\hline
\end{tabular}

Mixed linear model was used. Values are presented with mean $\pm S E$ (IC = pre-test vs. pos-test).

${ }^{+} p \leq 0.05$ (pre-test vs. pre-test), ${ }^{\# \# \#} p \leq 0.001$ (pos-test vs. pos-test); ${ }^{* *} p \leq 0.01,{ }^{* * *} p \leq 0.001$ (pre-test vs. pos-test).

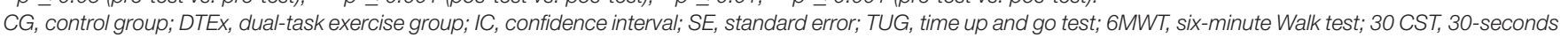
chair stand test.

Main effects of Group was detected for CERAD Recognition Memory $\left[F_{(1.63)}=6.888, p=0.01\right]$ and main effects of Time were detected on Immediate memory $\left[F_{(1 \cdot 70)}=38.534\right.$, $p<0.001]$ and Evocation memory $\left[F_{(1.69)}=16.309, p<0.001\right]$. A significant Group $\times$ Time interaction was detected only for CERAD Recognition Memory $\left[F_{(1.63)}=4.258, p=0.043\right]$. Bonferroni posttest analyses demonstrated that DTEx $(p<0.001)$ and CG $(p=0.005)$ increased the number of words on Immediate memory, however, only the DTEx group had improved performance on Evocation memory $(p<0.001)$ and Recognition $(p=0.006)$ (Table 2).

ANCOVA was applied-based on $z$-scores with adjustments by age, education, and sex-to test the hypothesis that the intervention effect sizes of DTEx could be higher than that of CG. No differences were found in Effects Size ( $Z$ score) between groups for Episodic memory (PAL TTA-DTEx $=0.632 \pm 0.137$, $\mathrm{CG}=0.259 \pm 0.161, p=0.085$ ), Sustained attention (RVP ML-DTEx $=0.464 \pm 0.126, C G=0.157 \pm 0.138, p=0.105)$, Immediate memory $(\mathrm{DTEx}=0.831 \pm 0.167, \mathrm{CG}=0.402 \pm 0.192$, $p=0.098)$, and Evocation memory $(\mathrm{DTEx}=0.511 \pm 0.159$, $\mathrm{CG}=0.314 \pm 0.186, p=0.424$ ).

DTEx group improvements showed moderate effect sizes on PAL SC (DTEx $=0.475 \pm 0.126, C G=0.064 \pm 0.148, p=0.039)$, PAL NPS (DTEx $=0.532 \pm 0.114, C G=0.093 \pm 0.132$, $p=0.015)$ and Recognition memory (DTEx $=0.468 \pm 0.147$, CG $=-0.060 \pm 0.169, p=0.022$ ), demonstrating significantly 


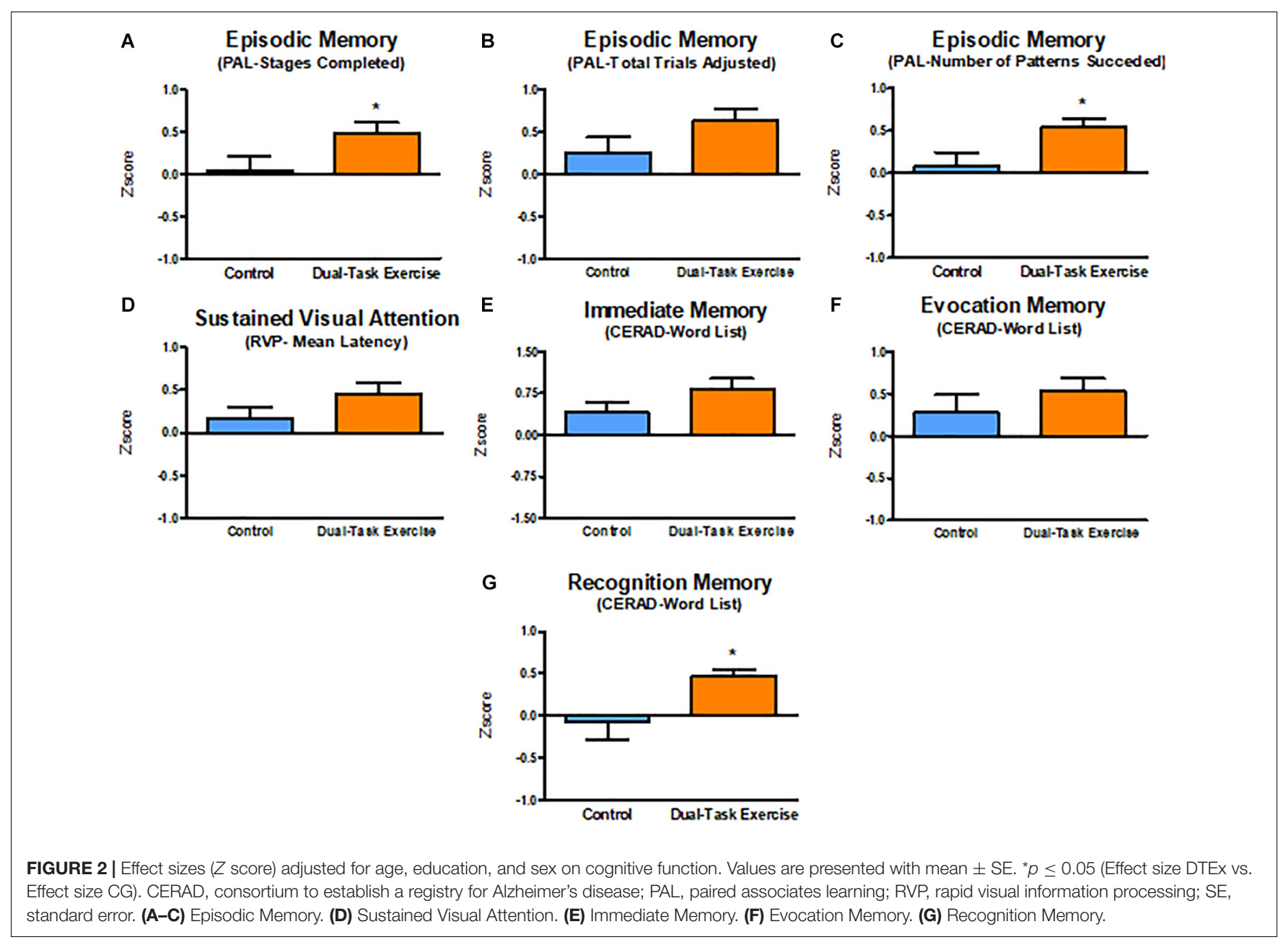

greater benefits of the intervention on episodic memory and recognition memory. Figure 2 illustrates effect size comparisons between groups on cognitive performance.

\section{Functional Exercise Capacity Results}

Main effects of Time and Group, as well Group $\times$ Time interaction were detected for functional mobility [Time: $F_{(1.61)}=7.587, p=0.008$; Group: $F_{(1.61)}=13.968, p<0.001$; interaction: $\left.F_{(1.61)}=8.970, p=0.004\right]$, lower limbs strength resistance [Time: $F_{(1.62)}=42.399, p<0.001$; Group: $F_{(1.62)}=25.296, p<0.001$; interaction: $F_{(1.62)}=16.570$, $p<0.001]$. Significant main effects of Group and Group $\times$ Time interaction for dual-task Agility [Group: $F_{(1.58)}=20.406$, $p<0.001$; interaction: $\left.F_{(1.58)}=8.315, p=0.006\right]$.

Cardiorespiratory fitness improvements showed the main effects of Group $\left[F_{(1.59)}=12.273, p=0.001\right]$ and Group $\times$ Time interaction $\left[F_{(1.59)}=14.265, p<0.001\right]$. Post hoc analyses indicated that the DTEx group showed significant improvements in functional exercise capacity on functional mobility $(p<0.001)$, lower limb strength resistance $(p<0.001)$, agility $(p<0.001)$, and cardiorespiratory fitness $(p<0.001)$. There were no differences from baseline for the CG (functional mobility: $p=0.876$; lower limb strength resistance: $p=0.114$; agility: $p=0.613$; cardiorespiratory fitness: $p=0.085$ ) (Table 3).

Analysis of dual-task performance were assessed by the Walking While Talking Test. Main effects of Group $\left[F_{(1.58)}=20.406, p<0.001, \eta^{2}=0.260\right]$ and a Group $\times$ Time interaction $\left[F_{(1.58)}=8.315, p=0.006, \eta^{2}=0.125\right]$ were detected for dual-task agility. Post hoc analysis indicated significant improvements for the DTEx group on walking speed $($ Pre $=1.192 \pm 0.043$, Post $=1.320 \pm 0.039, p=0.039)$. No significant effects were detected for the cognitive component [Verbal fluency-Time: $F_{(1.57)}=2.471, p=0.122$; Group: $F_{(1.57)}=1.750, p=0.191$; interaction: $F_{(1.57)}=1.115$, $p=0.295]$. These results indicate that after the intervention walking speed along with performance of a verbal fluency task was increased without declines on cognitive performance for the DTEx group. In contrast, the CG showed a reduction in dual-task agility $($ Pre $=1.020 \pm 0.054$, Post $=0.966 \pm 0.050$, $p=0.05)$ (Figure 3).

The DTEx group showed moderate effect sizes that were significantly greater than the CG for cardiorespiratory fitness (DTEx $=0.548 \pm 0.184$, CG $=-0.290 \pm 0.229$, $p=0.006$ ), functional mobility (DTEx $=0.704 \pm 0.142$, $\mathrm{CG}=-0.090 \pm 0.170, p=0.001)$, dual-task agility 


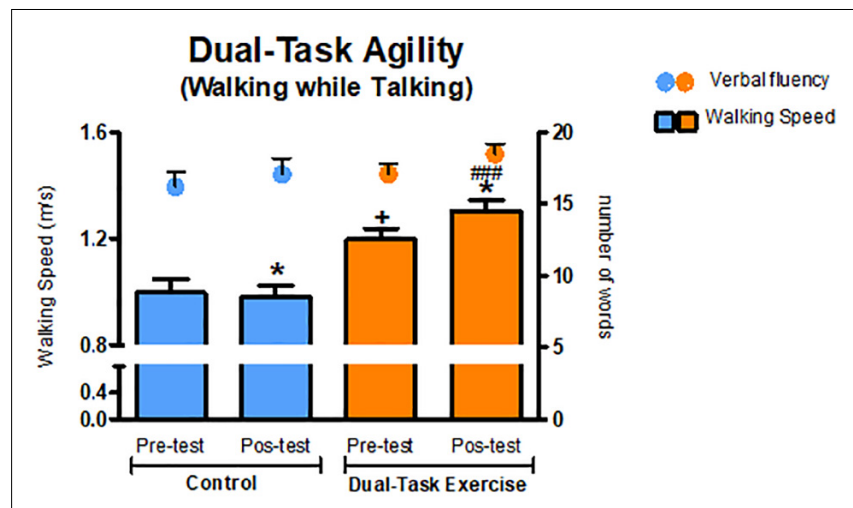

FIGURE 3 | Dual task performance (Walking while Talking Test). Values presented as mean \pm SE. ${ }^{+} p \leq 0.05$ (pre-test vs. pre-test); ${ }^{\# \#} p \leq 0.001$ (pos-test vs. pos-test); ${ }^{*} p \leq 0.05$ (pre-test vs. pos-test). SE, standard error.

$(\mathrm{DTEx}=0.463 \pm 0.146, \mathrm{CG}=-0.092 \pm 0,185, p=0.022)$, and agility $(\mathrm{DTEx}=0.653 \pm 0.134, \mathrm{GC}=0.209 \pm 0.167, p=0.043)$. The DTEx group showed a large effect size that was significantly greater than the CG for measurements of increased lower limb strength resistance $(\mathrm{DTEx}=1.188 \pm 0.148, \mathrm{CG}=0.368 \pm 0.173$, $p=0.001)$. Taken together, these results show a positive impact of the dual-task intervention program on several physical function and functional capacity measures (Figure 4).

\section{Quality of Life Results}

Main effects of Time were detected for physical functioning $\left[F_{(1.59)}=7.246, p=0.009\right]$ and vitality $\left[F_{(1,53)}=5.782\right.$, $p=0.020]$ Quality of Life (QoL) domains. Effects of Time and Group [Time: $F_{(1.54)}=11.275, p=0.001$; Group: $F_{(1.54)}=5.541$, $p=0.022]$ were detected on Limitations due to Physical Problems and General Health Perception domains [Time: $F_{(1.56)}=5.273$, $p=0.025$; Group: $F_{(1.56)}=4.050, p=0.049$ ].

The DTEx group improved in QoL related to Physical Functioning (DTEx: Pre $=75.946 \pm 3.184$, Post $=85.405 \pm$ 2.251, $p=0.001$ ), Role Limitations due to Physical Problems (DTEx: Pre $=70.312 \pm 7.134$, Post $=96.094 \pm 4.985$, $p=<0.001$ ), Vitality (DTEx: Pre $=67.000 \pm 3.123$, Post $=75.189 \pm 3.135, p=0.015)$, and General Health Perception (DTEx: Pre $=74.588 \pm 2.981$, Post $=82.441 \pm 2.482, p=0.018$ ). No statistical differences were found for changes in the CG on Quality of Life assessments.

\section{DISCUSSION}

In this paper we analyzed older adults' improvements on cognitive and functional capacity performances and quality of life in response to a dual-task exercise training intervention. We found there was a significant improvement in each assessment in the dual-task group after the intervention period. In contrast, the control group did not show any significant improvement in these tests. We suggest that 75 min of simultaneous multisensory cognitive stimulation and multimodal physical exercise in a dual-task paradigm, practiced regularly twice a week, may be effective at improving cognitive performance and preserving physical conditions that typically deteriorate in aging.

The hippocampus and the prefrontal cortex are affected during aging, and this may impair encoding and retrieval of episodic memory, working memory, and attention (Samson and Barnes, 2013). In contrast, regular physical exercise benefits brain structure and function, especially in aging populations, with changes occurring at molecular/cellular levels, brain structure/function, mental states, and higher order behavioral levels (Stillman et al., 2020), all of which improves functional losses (Erickson et al., 2019). Consistent with these prior results, our analysis revealed that 3 months of multimodal physical exercise training and multisensory cognitive stimulation in a dual-task paradigm improved cognitive performance on visual episodic memory, verbal episodic memory, sustained visual attention, and also improved functional capacity on cardiorespiratory conditioning, lower limb strength resistance, functional mobility, gait speed on a single task, dual-task performance and quality of life. Additionally, we estimated effect sizes ( $Z$ scores) to describe the proportional impact that the intervention had on cognitive and physical performance. The advantage of the $z$ score as we performed is that each subject (repeated measures) has a score based on how many standard deviations the outcome has changed from baseline for each participant. These results indicated that the intervention had significantly greater moderate effects on episodic visual memory (PAL SC, PAL NPS) and verbal recognition memory (List of words) compared to the CG, while the effects of the intervention on visual attention (RVP ML), immediate verbal memory and evocation verbal memory (word list) were not different from the CG.

It is important to emphasize that few clinical trials employing dual-task training have reported effect sizes in this fashion (Desjardins-Crepeau et al., 2016; Murillo-Garcia et al., 2020). Thus, our results add to this literature an estimate for how well the intervention program worked (Cordes et al., 2019), and clinically relevant information for the efficacy of the intervention program (Brown and Ezekowitz, 2017).

It has been described that visual episodic memory and verbal episodic memory impairments are sensitive markers to identify risk for dementia (Weissberger et al., 2017) and improvements in these functions may influence daily functions necessary for autonomy (Dikmen et al., 2014; Cansino et al., 2018). Our findings demonstrate that cognitive stimulation and multimodal exercise training in a dual-task format, $75 \mathrm{~min}$, twice a week, for 3 months, may have clinical importance for reducing age related cognitive decline. It has been suggested that while physical activity acts to preserve neuronal structural integrity (hardware), cognitive stimulation works on physiology and plasticity of neural circuits (software) and from these combined actions, cognitive function is amplified and prevent age-related cognitive decline and dementia (Cheng, 2016; Perneczky, 2019).

However, differently from the results shown in the present study, some studies have not found benefits using dualtask protocols (Legault et al., 2011; Ansai et al., 2017; 


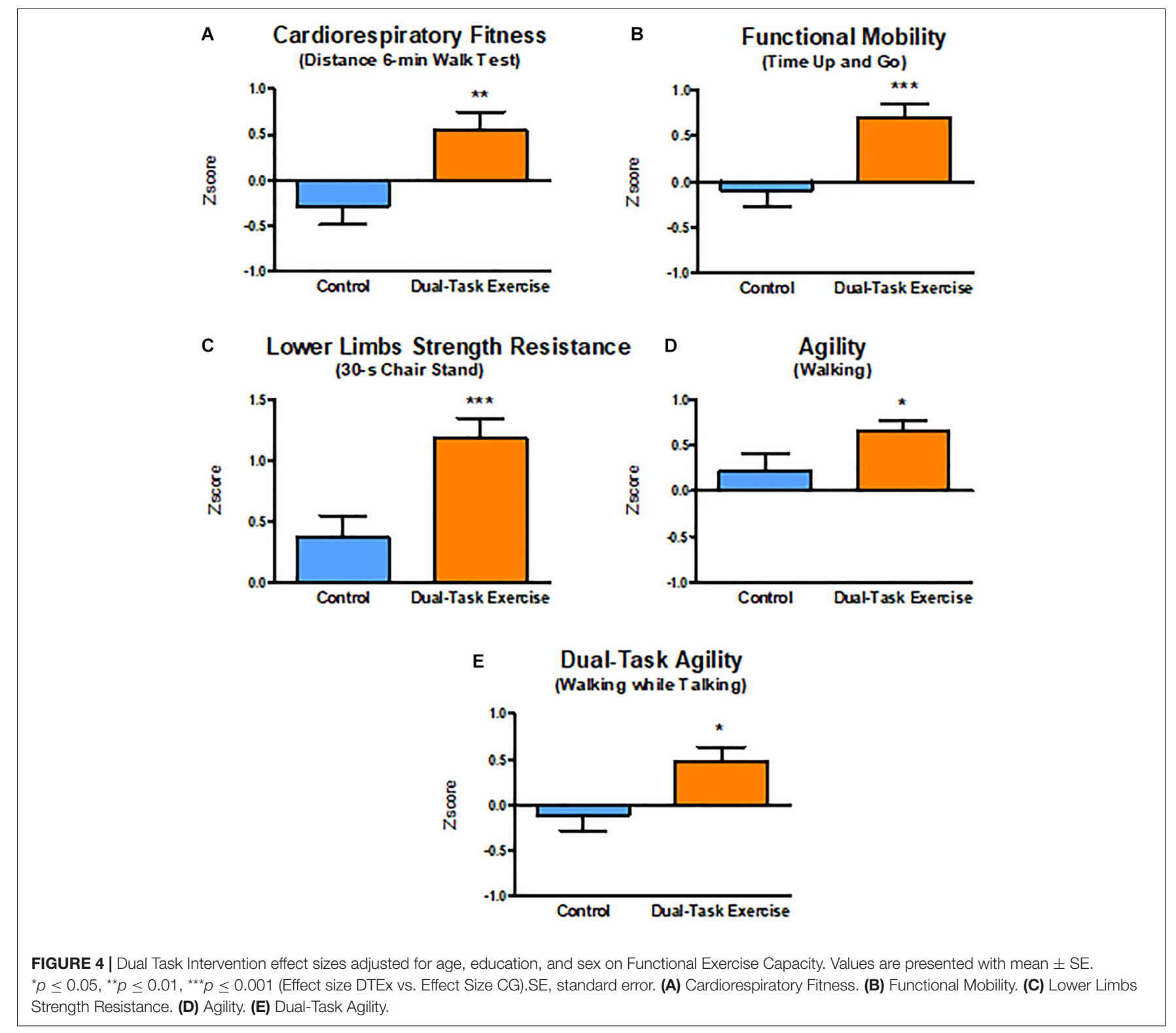

Boa Sorte Silva et al., 2018). The absence of results may be related to the different protocol characteristics such as exercise intensity, frequency, and duration (Gheysen et al., 2018; Stojan and Voelcker-Rehage, 2019; Wollesen et al., 2020). These components seem to mediate the relationship with cognitive performance (Guo et al., 2020). Medium (12-23 weeks) or short ( $<12$ weeks) interventions appear to be more effective in promoting cognitive improvements than longer interventions ( $\geq 24$ weeks), as well as lower frequencies ( $\leq 3$ session/week) compared to higher frequencies (Guo et al., 2020). However, it should be considered that some studies, even with similar duration and frequencies, present different results (Wollesen et al., 2020), which has led increasingly to consider the content of the intervention itself, such as the type of exercise and the complexity of the cognitive task.
It is also important to highlight that few studies have investigated the influence of intervention protocols on cognitive and physical domains simultaneously (Kitazawa et al., 2015; Gregory et al., 2017; Morita et al., 2018). However, considering that aging influences multiple systems and compromises the organism in a systemic way (He and Sharpless, 2017), the investigation of the physical and cognitive components are essential for the construction of programs that guarantee older adults' autonomy (Desjardins-Crepeau et al., 2016; Tait et al., 2017; Sipilä et al., 2018; Rezola-Pardo et al., 2019). While some protocols of physical multimodal interventions including strength and balance training seem to improve components of physical performance (Kang et al., 2015), it remains inconclusive whether they also improve cognitive performance (Ansai et al., 2017; Bruderer-Hofstetter et al., 2018). More recently, the influence of dual-task training on both has been investigated, 
identifying benefits for cognition, but not for lower limb strength or functional mobility (Morita et al., 2018). Thus, it remains important from the point of view of construction of intervention programs using cognitive and physical stimuli in dual-tasks, to consider the benefits of the multiple dimensions of functional declines associated with aging.

In the present study, all participants had not practiced physical exercise for at least 6 months before the intervention, and during physical training had their heart rate continuously and objectively monitored, to ensure that they were meeting the recommendations of exercise intensity. We also ensured that cognitive training was sufficiently challenging and included training with episodic memory, executive function, requiring functional responses to multisensory stimuli (Lauenroth et al., 2016; Tait et al., 2017). For this, we adopted a comprehensive cognitive stimulation program previously validated (De Oliveira et al., 2014), to stimulate language, attention, decision making, episodic memory, and a variety of functional responses to multisensory stimuli.

Although we strictly followed most of the recommendations for older adult assessment (Chodzko-Zajko et al., 2009; Gregory et al., 2013; Northey et al., 2018), some cognitive skills such as sustained visual attention and immediate and delayed verbal episodic memory (Word List), did not show effect sizes significantly different from the control group, suggesting that the observed effects might not be clinically significant. In addition, we observed, as previously mentioned (Calamia et al., 2012; Scharfen et al., 2018; Adcock et al., 2020), potential practice effects, where the control group seemed to improve on measures of immediate verbal memory.

In fact, it has been shown possible practice effects resulting from the test-retest design (Jutten et al., 2020). Our results, however, demonstrated significant interaction effects for episodic memory (PAL SC) and recognition memory (Word list-CERAD) suggesting that the intervention may improve these cognitive functions. Indeed, we observed that the interaction effects were associated with different performances over time in control and dual task group. As compared with control group, the dual task group matched by age, education, and physical activity level, subjected to evaluation and revaluation in the same time window with the same cognitive tests - showed better performance. Additionally, this observation reinforces the statistical approach using effect size measurements in the present report to ensure appropriate inferences.

Important to keep in mind that learning and social interaction effects in the aging brain facing new and novel challenging tasks may also play a role in the overall cognitive and quality of life changes. This confound factor would be related to the absence of a control group designed to assess possible influences of socialization on cognitive performance and quality of life. Indeed, a recent meta-analysis based on longitudinal studies indicated that high engagement in social activity and large social networks were (weakly) associated with better late-life cognitive function (Evans et al., 2019). Thus, more randomized clinical trials are needed to investigate the potential of causal effects of socialization on cognition and clarify this confound effect (Kuiper et al., 2016).
Intervention programs using cognitive and physical exercise in dual-task paradigms seem to improve performance in functional tasks of daily life in which divided attention between tasks is required (Smith et al., 2016; Wollesen and VoelckerRehage, 2019). Consistent with these findings, our results revealed significant improvements when performing a test that mimics situations of daily life, in which participants should walk and speak concurrently (Dual task test "Walking While Talking"). The results showed a significant increase in the average speed used for walking in the post intervention period, whereas the control group worsened after 3 months. These results have clinical repercussions, since worse performance in dual-task situations is strongly associated with an increased risk of falls and losses of functional independence (Beauchet et al., 2009; Smith et al., 2016). Recently, this worse performance has been identified as a risk for cognitive decline and dementia (Cullen et al., 2019; Rosso et al., 2019; Åhman et al., 2019).

Gait speed is considered an important clinical parameter for geriatric evaluation (Garcia-Pinillos et al., 2016; Inzitari et al., 2017). Low gait speed is a risk factor for falls, morbidity, institutionalization, and mortality (Liu et al., 2016; Perera et al., 2016). In addition, significant increases in the usual walking speed are associated with improved survival in community dwelling, healthy older adults, with significant increases in survival with each $0.1 \mathrm{~m} / \mathrm{sec}$ increase in speed (Studenski et al., 2011). The results in this study revealed that there was an increase of $13 \%$ in walking speed during a single task and $10 \%$ in a dual-task condition, suggesting that the participants increased their capacity to walk and perform a language task simultaneously.

It is worth mentioning that although the physical tests used in this study are simple, they assess habitual daily functions, which in turn are indicative of many underlying physiological processes (Jones et al., 1999; Ferguson-Stegall et al., 2017). The improvements observed in cardiorespiratory fitness, resistance of the lower limb muscle strength, functional mobility, and gait speed in dual-tasks contribute to the functional independence of older adults and are associated with quality of life (Gordon et al., 2020; Yang et al., 2020).

In previous reports (Cabral Soares et al., 2015; BentoTorres et al., 2017; Cabeça et al., 2018) we standardized CANTAB tests procedures. Although we do not yet have a large sample to define norms and cutoff points for the Brazilian population, CANTAB tests are largely independent of cultural differences which allows appropriate comparative analysis across countries (Robbins et al., 1994; De Luca et al., 2003; Lee et al., 2013; McPhee et al., 2013). Indeed, our findings agree with a number of previous studies from other countries using CANTAB (Robbins et al., 1994, 1998; De Luca et al., 2003; Smith et al., 2013) that confirm age related cognitive decline in selected tests for both temporal and prefrontal lobe functions.

Preventive interventions dedicated to healthy older adults are important to decrease the risk of cognitive decline or dementia (Cheng, 2016). Physical exercise and/or cognitive training during life are associated with neuroprotection through 
an increased cognitive reserve (Stern, 2012; Perneczky, 2019). This effect may postpone or attenuate clinical manifestations in case of disease, contributing to sustained functionality and independence (Gavelin et al., 2020). In addition, it is known that high costs on dual-tasks have been associated with increased risk of developing dementia (Cullen et al., 2019; Rosso et al., 2019) and that dual-task training protocols are necessary to improve performance (Wollesen and Voelcker-Rehage, 2013). Our results showed that participants in the Dual-Task intervention group improved cognitive and physical functioning, quality of life and dual-task performance after a multimodal physical-cognitive dual-task training intervention. Our approach was designed to be reliable, effective, and feasible to be included in the primary care or seniors' centers context, in group sessions, with limited and low-cost specific material resources.

We demonstrated that 3 months of dual-task multimodal physical exercise, $75 \mathrm{~min} / 2$ days per week, at moderate intensity, improves cognition, functional mobility, cardiorespiratory conditioning, upper and lower limbs strength, quality of life and dual-task performance on healthy older adults. Further investigations including intervention groups with a single stimulation, either physical training or cognitive training, for comparative purposes and blinding and randomized protocols will be necessary. In addition, longitudinal studies to investigate the detraining effects are also needed.

\section{DATA AVAILABILITY STATEMENT}

The raw data supporting the conclusions of this article will be made available by the authors, without undue reservation.

\section{ETHICS STATEMENT}

The studies involving human participants were reviewed and approved by Health Science Institute of Federal University of Pará Review Board (CAAE no 03427318.3.0000.0018). The patients/participants provided their written informed consent to participate in this study.

\section{REFERENCES}

Adcock, M., Fankhauser, M., Post, J., Lutz, K., Zizlsperger, L., Luft, A. R., et al. (2020). Effects of an in-home multicomponent exergame training on physical functions, cognition, and brain volume of older adults: a randomized controlled trial. Front. Med. 6:321. doi: 10.3389/fmed.2019.00321

American Thoracic Society (2002). ATS statement. Am. J. Respir. Crit. Care Med. 166, 111-117. doi: 10.1164/ajrccm.166.1.at1102

Ansai, J. H., de Andrade, L. P., de Souza Buto, M. S., Barroso, V. A., Farche, A. C. S., Rossi, P. G., et al. (2017). Effects of the addition of a dual task to a supervised physical exercise program on older adults' cognitive performance. J. Aging Phys. Activ. 25, 234-239. doi: 10.1123/japa.2016-0094

Beauchet, O., Annweiler, C., Dubost, V., Allali, G., Kressig, R. W., Bridenbaugh, S., et al. (2009). Stops walking when talking: a predictor of falls in older adults? Eur. J. Neurol. 16, 786-795. doi: 10.1111/j.1468-1331.2009.02612.x

Benedetti, T. B., Mazo, G. Z., and de Barros, M. V. G. (2004). Application of the international physical activity questionnaire (IPAQ) for evaluation of elderly

\section{AUTHOR CONTRIBUTIONS}

CD and NB-T participated in the conception of the study, guidance, statistical analysis, data analysis, interpretation of results, and writing. JB-T contributed to the conception of the study, statistical analysis, data analysis, interpretation of results, formatting of graphics, and writing. VC participated in data collection, intervention program, data tabulation, and formatting adjustments in the text. MS contributed to writing and adjustments in the text. JC and HP contributed on data collection, tabulation, and intervention program. AT participated in data collection and tabulation. NJ participated in data collection, data tabulation, intervention program, statistical analysis, and writing. KE contributed to interpretation of results and writing. All authors have read and approved the manuscript final version and agreed with the order of presentation of the authors.

\section{FUNDING}

CWPD was supported by PROCAD-AMAZÔNIA, Grant/Award Number: 88881.370785/2019-01. Authors was supported by PROPESP/UFPA. This study was supported by Fundação de Amparo à Pesquisa do Pará-FADESP/Pró-Reitoria de Pesquisa e Pós-Graduação da Universidade Federal do Pará-PROPESP Edital 2019-PIAPA; Coordenação de Aperfeiçoamento de Pessoal de Nível Superior-CAPES-Pró-Amazônia, Grant No. 3311/2013; Brazilian Research Council-CNPq Grant No: 307749/2004-5 and 471077/2007-0 for CD, Fundação Amazônia de Amparo a Estudos e Pesquisas do Pará-FAPESPA, ICAAF No 039/2017; and Programa Institucional de Bolsas de Iniciação Científica e de Desenvolvimento Tecnológico e Inovação-PROPESP Edital $06 / 2019$.

\section{ACKNOWLEDGMENTS}

We would like to thank all participants for collaboration. In addition, we would like to thank all community centers and public health units that made this work possible.

women: concurrent validity and test-retest reprodutibility. R. Bras. Ci. Mov. 12, 25-34. doi: 10.18511/rbcm.v12i1.538

Bento-Torres, N. V. O., Bento-Torres, J., Tomás, A. M., Costa, V. O., Corrêa, P. G. R., Costa, C. N. M., et al. (2017). Influence of schooling and age on cognitive performance in healthy older adults. Braz. J. Med. Biol. Res. 50:e5892. doi: 10.1590/1414-431X201 65892

Bertolucci, P. H. F., Brucki, S. M. D., Campacci, S. R., and Juliano, Y. (1994). O mini-exame do estado mental em uma população geral: impacto da escolaridade. Arq. Neuropsiquiatr. 52, 1-7. doi: 10.1590/S0004282X1994000100001

Bertolucci, P. H. F., Okamoto, I. H., Brucki, S. M. D., Siviero, M. O., Neto, J. T., and Ramos, L. R. (2001). Applicability of the CERAD neuropsychological battery to Brazilian elderly. Arq. Neuropsiquiatr. 59, 532-536. doi: 10.1590/S0004282X2001000400009

Bertolucci, P. H. F., Okamoto, I. H., Neto, J. T., Ramos, L. R., and Brucki, S. M. D. (1998). Desempenho da população brasileira na bateria neuropsicológica do 
Consortium to Establish a Registry for Alzheimer's Disease (CERAD). Rev. Psiquiatr. Clin. 25, 80-83.

Boa Sorte Silva, N. C., Gill, D. P., Owen, A. M., Liu-Ambrose, T., Hachinski, V., Shigematsu, R., et al. (2018). Cognitive changes following multiple-modality exercise and mind-motor training in older adults with subjective cognitive complaints: the M4 study. PLoS One 13:e0196356. doi: 10.1371/journal.pone. 0196356

Brown, P. M., and Ezekowitz, J. A. (2017). Composite end points in clinical trials of heart failure therapy. Circ. Heart Fail. 10:e003222. doi: 10.1161/ CIRCHEARTFAILURE.116.003222

Bruderer-Hofstetter, M., Rausch-Osthoff, A. K., Meichtry, A., Münzer, T., and Niedermann, K. (2018). Effective multicomponent interventions in comparison to active control and no interventions on physical capacity, cognitive function and instrumental activities of daily living in elderly people with and without mild impaired cognition-a systematic revi. Ageing Res. Rev. 45, 1-14. doi: 10.1016/j.arr.2018.04.002

Cabeça, H. L. S., Rocha, L. C., Sabbá, A. F., Tomás, A. M., Bento-Torres, N. V. O., Anthony, D. C., et al. (2018). The subtleties of cognitive decline in multiple sclerosis: an exploratory study using hierarchichal cluster analysis of CANTAB results. BMC Neurol. 18:140. doi: 10.1186/s12883-018-1141-1

Cabral Soares, F., de Oliveira, T. C. G., Macedo, L. D. D., Diniz, D. L. W. P., BentoTorres, N. V. O., Bento-Torres, J., et al. (2015). CANTAB object recognition and language tests to detect aging cognitive decline: an exploratory comparative study. Clin. Interv. Aging 10, 37-48. doi: 10.2147/CIA.S68186

Cadore, E. L., and Izquierdo, M. (2013). How to simultaneously optimize muscle strength, power, functional capacity, and cardiovascular gains in the elderly: an update. Age 35, 2329-2344. doi: 10.1007/s11357-012-9503-x

Calamia, M., Markon, K., and Tranel, D. (2012). Scoring higher the second time around: meta-analyses of practice effects in neuropsychological assessment. Clin. Neuropsychol. 26, 543-570. doi: 10.1080/13854046.2012. 680913

Cambridge Cognition (2016). CANTAB Insight: A Scientific and Clinical Review. Cambridge: Cambridge Cognition.

Cansino, S., Torres-Trejo, F., Estrada-Manilla, C., Hernández-Ramos, E., Martínez-Galindo, J. G., Gómez-Fernández, T., et al. (2018). Mediators of episodic memory decay across the adult life span. Sci. Rep. 8:2610. doi: 10.1038/ s41598-018-20884-2

Cansino, S., Torres-Trejo, F., Estrada-Manilla, C., Pérez-Loyda, M., RamírezBarajas, L., Hernández-Ladrón-deGuevara, M., et al. (2020). Predictors of working memory maintenance and decline in older adults. Arch. Gerontol. Geriatr. 89:104074. doi: 10.1016/j.archger.2020.104074

Cheng, S. T. (2016). Cognitive reserve and the prevention of dementia: the role of physical and cognitive activities. Curr. Psychiatry Rep. 18:85. doi: 10.1007/ s11920-016-0721-2

Chodzko-Zajko, W. J., Proctor, D. N., Fiatarone Singh, M. A., Minson, C. T., Nigg, C. R., Salem, G. J., et al. (2009). Exercise and physical activity for older adults. Med. Sci. Sports Exerc. 41, 1510-1530. doi: 10.1249/MSS.0b013e3181a0c95c

Ciconelli, R. M., Mb Ferraz, Santos, W., Meinao, I., and Quaresma, M. R. (1999). Traducao para a lingua Portuguesa e validacao do questionario generico de avaliacao de qualidade de vida SF-36 (Brasil SF-36). Rev. Bras. Reumatol. 39, $143-150$.

Coelho, L., Hauck, K., McKenzie, K., Copeland, J. L., Kan, I. P., Gibb, R. L., et al. (2020). The Association between sedentary behavior and cognitive ability in older adults. Aging Clin. Exp. Res. 32, 2339-2347. doi: 10.1007/s40520-01901460-8

Cohen, J. (1992). A power primer. Psychol. Bull. 112, 155-159. doi: 10.1037/00332909.112.1.155

Colcombe, S., and Kramer, A. F. (2003). Fitness effects on the cognitive function of older adults. Psychol. Sci. 14, 125-130. doi: 10.1111/1467-9280.t01-101430

Cognition, C. (2014). Cantab Research Suite version 6 - Test Administration Guide. Cambridge Cognition Limited, 1-309.

Cordes, T., Bischoff, L. L., Schoene, D., Schott, N., Voelcker-Rehage, C., Meixner, C., et al. (2019). A multicomponent exercise intervention to improve physical functioning, cognition and psychosocial well-being in elderly nursing home residents: a study protocol of a randomized controlled trial in the PROCARE (Prevention and Occupational Health in Long-T. BMC Geriatr. 19:369. doi: 10.1186/s12877-019-1386-6
Cullen, S., Borrie, M., Carroll, S., Sarquis-Adamson, Y., Pieruccini-Faria, F., McKay, S., et al. (2019). Are cognitive subtypes associated with dual-task gait performance in a clinical setting? J. Alzheimers Dis. 71, S57-S64. doi: 10.3233/ JAD- 181196

De Jager, C. A., Milwain, E., and Budge, M. (2002). Early detection of isolated memory deficits in the elderly: the need for more sensitive neuropsychological tests. Psychol. Med. 32, 483-491. doi: 10.1017/S003329170200524X

De Luca, C. R., Wood, S. J., Anderson, V., Buchanan, J. A., Proffitt, T. M., Mahony, K., et al. (2003). Normative data from the CANTAB. I: development of executive function over the lifespan. J. Clin. Exp. Neuropsychol. 25, 242-254. doi: 10.1076/ jcen.25.2.242.13639

De Oliveira, T. C., Soares, F. C., De Macedo, L. D., Diniz, D. L., Bento-Torres, N. V., and Picanço-Diniz, C. W. (2014). Beneficial effects of multisensory and cognitive stimulation on age-related cognitive decline in long-term-care institutions. Clin. Interv. Aging 9, 309-320. doi: 10.2147/CIA.S54383

Desjardins-Crepeau, L., Berryman, N., Fraser, S., Vu, T. T., Kergoat, M. J., Li, K. Z., et al. (2016). Effects of combined physical and cognitive training on fitness and neuropsychological outcomes in healthy older adults. Clin. Interv. Aging 11, 1287-1299. doi: 10.2147/CIA.S115711

Dikmen, S. S., Bauer, P. J., Weintraub, S., Mungas, D., Slotkin, J., Beaumont, J. L., et al. (2014). Measuring episodic memory across the lifespan: NIH toolbox picture sequence memory test. J. Int. Neuropsychol. Soc. 20, 611-619. doi: 10. $1017 /$ S1355617714000460

Eggenberger, P., Theill, N., Holenstein, S., Schumacher, V., and de Bruin, E. D. (2015). Multicomponent physical exercise with simultaneous cognitive training to enhance dual-task walking of older adults: a secondary analysis of a 6month randomized controlled trial with I-year follow-up. Clin. Interv. Aging 10, 1711-1732. doi: 10.2147/CIA.S91997

Ekblom, M. M., Ekblom, ÖB., Börjesson, M., Bergström, G., Jern, C., and Wallin, A. (2019). Device-measured sedentary behavior, physical activity and aerobic fitness are independent correlates of cognitive performance in healthy middleaged adults-results from the SCAPIS pilot study. Int. J. Environ. Res. Public Health 16:5136. doi: 10.3390/ijerph16245136

Erickson, K. I., Hillman, C., Stillman, C. M., Ballard, R. M., Bloodgood, B., Conroy, D. E., et al. (2019). Physical activity, cognition, and brain outcomes: a review of the 2018 physical activity guidelines. Med. Sci. Sports Exerc. 51, 1242-1251. doi: 10.1249/MSS.0000000000001936

Evans, I. E. M., Martyr, A., Collins, R., Brayne, C., and Clare, L. (2019). Social isolation and cognitive function in later life: a systematic review and metaanalysis. J. Alzheimers Dis. 70, S119-S144. doi: 10.3233/JAD-180501

Falbo, S., Condello, G., Capranica, L., Forte, R., and Pesce, C. (2016). Effects of physical-cognitive dual task training on executive function and gait performance in older adults: a randomized controlled trial. Biomed. Res. Int. 2016:5812092. doi: 10.1155/2016/5812092

Ferguson-Stegall, L., Vang, M., Wolfe, A. S., and Thomsen, K. M. (2017). A 9-Week jaques-dalcroze eurhythmics intervention improves single and dual-task gait speed in community-dwelling older people. J. Phys. Act. Health 14, 740-744. doi: 10.1123/jpah.2017-0416

Forte, R., Boreham, C. A., Leite, J. C., De Vito, G., Brennan, L., Gibney, E. R., et al. (2013). Enhancing cognitive functioning in the elderly: multicomponent vs resistance training. Clin. Interve. Aging 8, 19-27. doi: 10.2147/CIA.S36514

Garber, C. E., Blissmer, B., Deschenes, M. R., Franklin, B. A., Lamonte, M. J., Lee, I. M., et al. (2011). Quantity and quality of exercise for developing and maintaining cardiorespiratory, musculoskeletal, and neuromotor fitness in apparently healthy adults: guidance for prescribing exercise. Med. Sci. Sports Exerc. 43, 1334-1359. doi: 10.1249/MSS.0b013e318213fefb

Garcia-Pinillos, F., Cozar-Barba, M., Munoz-Jimenez, M., Soto-Hermoso, V., and Latorre-Roman, P. (2016). Gait speed in older people: an easy test for detecting cognitive impairment, functional independence, and health state. Psychogeriatrics 16, 165-171. doi: 10.1111/psyg.12133

Garneau, L., and Aguer, C. (2019). Role of myokines in the development of skeletal muscle insulin resistance and related metabolic defects in type 2 diabetes. Diabetes Metab. 45, 505-516. doi: 10.1016/j.diabet.2019.02.006

Gavelin, H. M., Dong, C., Minkov, R., Bahar-Fuchs, A., Ellis, K. A., Lautenschlager, N. T., et al. (2020). Combined physical and cognitive training for older adults with and without cognitive impairment: a systematic review and network metaanalysis of randomized controlled trials. Ageing Res. Rev. 66:101232. doi: 10. 1016/j.arr.2020.101232 
Gheysen, F., Poppe, L., DeSmet, A., Swinnen, S., Cardon, G., De Bourdeaudhuij, I., et al. (2018). Physical activity to improve cognition in older adults: can physical activity programs enriched with cognitive challenges enhance the effects? A systematic review and meta-analysis. Int. J. Behav. Nutr. Phys. Act. 15:63. doi: 10.1186/s12966-018-0697-x

Gordon, S. J., Baker, N., Kidd, M., Maeder, A., and Grimmer, K. A. (2020). Pre-Frailty factors in community-dwelling 40-75 year olds: opportunities for successful ageing. BMC Geriat. 20:96. doi: 10.1186/s12877-020-1490-7

Green, D. J., Hopman, M. T., Padilla, J., Laughlin, M. H., and Thijssen, D. H. (2017). Vascular adaptation to exercise in humans: role of hemodynamic stimuli. Physiol. Rev. 97, 495-528. doi: 10.1152/physrev.00014.2016

Gregory, M. A., Boa Sorte Silva, N. C., Gill, D. P., McGowan, C. L., Liu-Ambrose, T., Shoemaker, J. K., et al. (2017). Combined dual-task gait training and aerobic exercise to improve cognition, mobility, and vascular health in communitydwelling older adults at risk for future cognitive decline1. J. Alzheimers Dis. 57, 747-763. doi: 10.3233/JAD-161240

Gregory, M. A., Gill, D. P., and Petrella, R. J. (2013). Brain health and exercise in older adults. Curr. Sports Med. Rep. 12, 256-271. doi: 10.1249/JSR. 0b013e31829a74fd

Guo, W., Zang, M., Klich, S., Kawczyński, A., Smoter, M., and Wang, B. (2020). Effect of combined physical and cognitive interventions on executive functions in older adults: a meta-analysis of outcomes. Int. J. Environ. Res. Public Health 17:6166. doi: 10.3390/ijerph17176166

Hall, C. D., Echt, K. V., Wolf, S. L., and Rogers, W. A. (2011). Cognitive and motor mechanisms underlying older adults' ability to divide attention while walking. Phys. Ther. 91, 1039-1050. doi: 10.2522/ptj.2010 0114

He, S., and Sharpless, N. E. (2017). Senescence in health and disease. Cell 169, 1000-1011. doi: 10.1016/j.cell.2017.05.015

Herold, F., Hamacher, D., Schega, L., and Müller, N. G. (2018). Thinking while moving or moving while thinking-concepts of motor-cognitive training for cognitive performance enhancement. Front. Aging Neurosci. 10:228. doi: 10. 3389/fnagi.2018.00228

Inzitari, M., Calle, A., Esteve, A., Casas, Á, Torrents, N., and Martínez, N. (2017). ¿Mides la velocidad de la marcha en tu práctica diaria? Una revisión. Rev. Esp. Geriatr. Gerontol. 52, 35-43. doi: 10.1016/j.regg.2015.12.010

Irwin, K., Sexton, C., Daniel, T., Lawlor, B., and Naci, L. (2018). Healthy aging and dementia: two roads diverging in midlife? Front. Aging Neurosci. 10:275. doi: $10.3389 /$ fnagi.2018.00275

Jones, C. J., Rikli, R. E., and Beam, W. C. (1999). A 30-s chair-stand test as a measure of lower body strength in community-residing older adults. Res. Q. Exerc. Sport 70, 113-119. doi: 10.1080/02701367.1999.10608028

Jutten, R. J., Grandoit, E., Foldi, N. S., Sikkes, S. A. M., Jones, R. N., Choi, S. E., et al. (2020). Lower practice effects as a marker of cognitive performance and dementia risk: a literature review. Alzheimers Dement. 12, 1-12. doi: 10.1002/ dad2.12055

Kang, S., Hwang, S., Klein, A. B., and Kim, S. H. (2015). Multicomponent exercise for physical fitness of community-dwelling elderly women. J. Phys. Ther. Sci. 27, 911-915. doi: 10.1589/jpts.27.911

Kitazawa, K., Showa, S., Hiraoka, A., Fushiki, Y., Sakauchi, H., and Mori, M. (2015). Effect of a dual-task net-step exercise on cognitive and gait function in older adults. J. Geriatr. Phys. Ther. 38, 133-140. doi: 10.1519/JPT.00000000000 00029

Kivimäki, M., Singh-Manoux, A., Pentti, J., Sabia, S., Nyberg, S. T., Alfredsson, L., et al. (2019). Physical inactivity, cardiometabolic disease, and risk of dementia: an individual-participant meta-analysis. BMJ (Online) 365:11495. doi: 10.1136/ bmj.11495

Kivipelto, M., Mangialasche, F., and Ngandu, T. (2018). Lifestyle interventions to prevent cognitive impairment, dementia and Alzheimer disease. Nat. Rev. Neurol. 14, 653-666. doi: 10.1038/s41582-0180070-3

Korkki, S. M., Richter, F. R., Jeyarathnarajah, P., and Simons, J. S. (2020). Healthy ageing reduces the precision of episodic memory retrieval. Psychol. Aging 35, 124-142. doi: 10.1037/pag0000432

Kuiper, J. S., Zuidersma, M., Zuidema, S. U., Burgerhof, J. G. M., Stolk, R. P., Oude Voshaar, R. C., et al. (2016). Social relationships and cognitive decline: a systematic review and meta-analysis of longitudinal cohort studies. Int. J. Epidemiol. 45:dyw089. doi: 10.1093/ije/dyw089
Lauenroth, A., Ioannidis, A. E., and Teichmann, B. (2016). Influence of combined physical and cognitive training on cognition: a systematic review. BMC Geriatr. 16:141. doi: 10.1186/s12877-016-0315-1

Lee, A., Archer, J., Wong, C. K. Y., Chen, S. H. A., and Qiu, A. (2013). Age-related decline in associative learning in healthy Chinese adults. PLoS One 8:e80648. doi: 10.1371/journal.pone.0080648

Legault, C., Jennings, J. M., Katula, J. A., Dagenbach, D., Gaussoin, S. A., Sink, K. M., et al. (2011). Designing clinical trials for assessing the effects of cognitive training and physical activity interventions on cognitive outcomes: the seniors health and activity research program pilot (SHARP-P) study, a randomized controlled trial. BMC Geriatr. 11:27. doi: 10.1186/1471-2318-11-27

Li, K. Z. H., Bherer, L., Mirelman, A., Maidan, I., and Hausdorff, J. M. (2018). Cognitive involvement in balance, gait and dual-tasking in aging: a focused review from a neuroscience of aging perspective. Front. Neurol. 9:913. doi: 10.3389/fneur.2018.00913

Liu, B., Hu, X., Zhang, Q., Fan, Y., Li, J., Zou, R., et al. (2016). Usual walking speed and all-cause mortality risk in older people: a systematic review and meta-analysis. Gait Posture 44, 172-177. doi: 10.1016/j.gaitpost.2015.12.008

Liu-Ambrose, T., and Donaldson, M. G. (2009). Exercise and cognition in older adults: is there a role for resistance training programmes? Br. J. Sports Med. 43, 25-27. doi: 10.1136/bjsm.2008.055616

Magnon, V., Vallet, G. T., and Auxiette, C. (2018). Sedentary behavior at work and cognitive functioning: a systematic review. Front. Public Health 6:239. doi: 10.3389/fpubh.2018.00239

McDonough, I. M., Wood, M. M., and Miller, W. S. (2019). A review on the trajectory of attentional mechanisms in aging and the Alzheimer's disease continuum through the attention network test. Yale J. Biol. Med. 92, 37-51.

McPhee, J. S., Hogrel, J. Y., Maier, A. B., Seppet, E., Seynnes, O. R., Sipilä, S., et al. (2013). Physiological and functional evaluation of healthy young and older men and women: design of the european myoage study. Biogerontology 14, 325-337. doi: 10.1007/s10522-013-9434-7

Morita, E., Yokoyama, H., Imai, D., Takeda, R., Ota, A., Kawai, E., et al. (2018). Effects of 2-year cognitive-motor dual-task training on cognitive function and motor ability in healthy elderly people: a pilot study. Brain Sci. 8:86. doi: 10 . 3390/brainsci8050086

Morris, J. N., Heady, J. A., Raffle, P. A. B., Roberts, C. G., and Parks, J. W. (1953). Coronary heart-disease and physical activity of work. Lancet 262, 1053-1057. doi: 10.1016/S0140-6736(53)90665-5

Murillo-Garcia, A., Villafaina, S., Collado-Mateo, D., Leon-Llamas, J. L., and Gusi, N. (2020). Effect of dance therapies on motor-cognitive dual-task performance in middle-aged and older adults: a systematic review and meta-analysis. Disabil. Rehabil. doi: 10.1080/09638288.2020.1735537 [Epub ahead of print].

Nichols, E., Szoeke, C. E. I., Vollset, S. E., Abbasi, N., Abd-Allah, F., Abdela, J., et al. (2019). Global, regional, and national burden of Alzheimer's disease and other Dementias, 1990-2016: a systematic analysis for the global burden of disease study 2016. Lancet Neurol. 18, 88-106. doi: 10.1016/S1474-4422(18) 30403-4

Ninot, G., Flori, N., Huteau, M.-E., Stoebner-Delbarre, A., and Senesse, P. (2020). Activités physiques et cancers: des bénéfices prouvés pendant et après les traitements. Bull. Cancer 107, 474-489. doi: 10.1016/j.bulcan.2019. 11.017

Northey, J. M., Cherbuin, N., Pumpa, K. L., Smee, D. J., and Rattray, B. (2018). Exercise interventions for cognitive function in adults older than 50: a systematic review with meta-analysis. Br. J. Sports Med. 52, 154-160. doi: 10.1136/bjsports-2016-096587

Owen, N., Healy, G. N., Dempsey, P. C., Salmon, J., Timperio, A., Clark, B. K., et al. (2020). Sedentary behavior and public health: integrating the evidence and identifying potential solutions. Annu. Rev. Public Health 41, 265-287. doi: 10.1146/annurev-publhealth-040119-094201

Perera, S., Patel, K. V., Rosano, C., Rubin, S. M., Satterfield, S., Harris, T., et al. (2016). Gait speed predicts incident disability: a pooled analysis. J. Gerontol. A Biol. Sci. Med. Sci. 71, 63-71. doi: 10.1093/gerona/glv126

Perneczky, R. (2019). Dementia prevention and reserve against neurodegenerative disease. Dialogues Clin. Neurosci. 21, 53-60. doi: 10.31887/DCNS.2019.21.1/ rperneczky2

Podsiadlo, D., and Richardson, S. (1991). The timed "Up \& Go": a test of basic functional mobility for frail elderly persons. J. Am. Geriatr. Soc. 39, 142-148. doi: 10.1111/j.1532-5415.1991.tb01616.x 
Prince, M., Wimo, A., Guerchet, M., Ali, G.-C., Wu, Y.-T., Prina, M., et al. (2015). World Alzheimer Report 2015- The Global Impact of Dementia an Analysis of Prevalence, Incidence, Cost and Trends. London: Alzheimer's Disease International. doi: 10.1111/j.0963-7214.2004.00293.x

Rezola-Pardo, C., Arrieta, H., Gil, S. M., Yanguas, J. J., Iturburu, M., Irazusta, J., et al. (2019). A randomized controlled trial protocol to test the efficacy of a dual-task multicomponent exercise program in the attenuation of frailty in long-term nursing home residents: aging-on dual-task study. BMC Geriatr. 19:6. doi: 10.1186/s12877-018-1020-z

Rezuş, E., Burlui, A., Cardoneanu, A., Rezuş, C., Codreanu, C., Pârvu, M., et al. (2020). Inactivity and skeletal muscle metabolism: a vicious cycle in old age. Int. J. Mol. Sci. 21:592. doi: 10.3390/ijms21020592

Robbins, T. W., James, M., Owen, A. M., Sahakian, B. J., Lawrence, A. D., Mcinnes, L., et al. (1998). A study of performance on tests from the CANTAB battery sensitive to frontal lobe dysfunction in a large sample of normal volunteers: implications for theories of executive functioning and cognitive aging. J. Int. Neuropsychol. Soc. 4, 474-490. doi: 10.1017/S1355617798455073

Robbins, T. W., James, M., Owen, A. M., Sahakian, B. J., McInnes, L., and Rabbitt, P. (1994). Cambridge neuropsychological test automated battery (CANTAB): a factor analytic study of a large sample of normal elderly volunteers. Dement. Geriatr. Cogn. Disord. 5, 266-281. doi: 10.1159/000106735

Rosso, A. L., Metti, A. L., Faulkner, K., Redfern, M., Yaffe, K., Launer, L., et al. (2019). Complex walking tasks and risk for cognitive decline in high functioning older adults. J. Alzheimers Dis. 71, S65-S73. doi: 10.3233/JAD181140

Sahakian, B. J., and Owen, A. M. (1992). Computerized assessment in neuropsychiatry using CANTAB: discussion paper. R. Soc. Med. 85, 399-402. doi: $10.1177 / 014107689208500711$

Samson, R. D., and Barnes, C. A. (2013). Impact of aging brain circuits on cognition. Eur. J. Neurosci. 37, 1903-1915. doi: 10.1111/ejn.12183.Impact

Scharfen, J., Blum, D., and Holling, H. (2018). Response time reduction due to retesting in mental speed tests: a meta-analysis. J. Intell. 6:6. doi: 10.3390/ jintelligence6010006

Sink, K. M., Espeland, M. A., Castro, C. M., Church, T., Cohen, R., Dodson, J. A., et al. (2015). Effect of a 24-month physical activity intervention vs health education on cognitive outcomes in sedentary older adults. JAMA 314:781. doi: 10.1001/jama.2015.9617

Sipilä, S., Tirkkonen, A., Hänninen, T., Laukkanen, P., Alen, M., Fielding, R. A., et al. (2018). Promoting safe walking among older people: the effects of a physical and cognitive training intervention vs. physical training alone on mobility and falls among older community-dwelling men and women (the PASSWORD study): design and methods of a randomize. BMC Geriatr. 18:215. doi: 10.1186/s12877-018-0906-0

Smith, E., Cusack, T., and Blake, C. (2016). The effect of a dual task on gait speed in community dwelling older adults: a systematic review and meta-analysis. Gait Posture 44, 250-258. doi: 10.1016/j.gaitpost.2015.12.017

Smith, P. J., Need, A. C., Cirulli, E. T., Chiba-Falek, O., and Attix, D. K. (2013). A comparison of the cambridge automated neuropsychological test battery (CANTAB) with "Traditional” neuropsychological testing instruments. J. Clin. Exp. Neuropsychol. 35, 319-328. doi: 10.1080/13803395.2013.771618

Stern, Y. (2012). Cognitive reserve in ageing and Alzheimer's disease. Lancet Neurol. 11, 1006-1012. doi: 10.1016/S1474-4422(12)70191-6

Stillman, C. M., Esteban-Cornejo, I., Brown, B., Bender, C. M., and Erickson, K. I. (2020). Effects of exercise on brain and cognition across age groups and health states. Trends Neurosci. 43, 1-11. doi: 10.1016/j.tins.2020. 04.010

Stojan, R., and Voelcker-Rehage, C. (2019). A systematic review on the cognitive benefits and neurophysiological correlates of exergaming in healthy older adults. J. Clin. Med. 8:734. doi: 10.3390/jcm8050734

Studenski, S., Perera, S., and Patel, K. (2011). Gait speed and survival in older adults. JAMA 305, 50-58. doi: 10.1001/jama.2010.1923.Gait

Tait, J. L., Duckham, R. L., Milte, C. M., Main, L. C., and Daly, R. M. (2017). Influence of sequential vs. simultaneous dual-task exercise training on cognitive function in older adults. Front. Aging Neurosci. 9:368. doi: 10.3389/fnagi.2017. 00368

Weissberger, G. H., Strong, J. V., Stefanidis, K. B., Summers, M. J., Bondi, M. W., and Stricker, N. H. (2017). Diagnostic accuracy of memory measures in Alzheimer's dementia and mild cognitive impairment: a systematic review and meta-analysis. Neuropsychol. Rev. 27, 354-388. doi: 10.1007/s11065-0179360-6

Wild, K., Howieson, D., Webbe, F., Seelye, A., and Kaye, J. (2008). The status of computerised cognitive testing in aging: a systematic review. Alzheimers Dement. 4, 428-437. doi: 10.1016/j.jalz.2008.07.003.The

Wollesen, B., and Voelcker-Rehage, C. (2013). Training effects on motor-cognitive dual-task performance in older adults. Eur. Rev. Aging Phys. Act. 11, 5-24. doi: 10.1007/s11556-013-0122-z

Wollesen, B., and Voelcker-Rehage, C. (2019). Differences in cognitive-motor interference in older adults while walking and performing a visual-verbal stroop task. Front. Aging Neurosci. 10:426. doi: 10.3389/fnagi.2018.00426

Wollesen, B., Wildbredt, A., van Schooten, K. S., Lim, M. L., and Delbaere, K. (2020). The effects of cognitive-motor training interventions on executive functions in older people: a systematic review and meta-analysis. Eur. Rev. Aging Phys. Act. 17:9. doi: 10.1186/s11556-020-00240-y

Yang, S., Li, T., Yang, H., Wang, J., Liu, M., Wang, S., et al. (2020). Association between muscle strength and health-related quality of life in a Chinese rural elderly population: a cross-sectional study. BMJ Open 10:e026560. doi: 10.1136/ bmjopen-2018-026560

Zhu, X., Yin, S., Lang, M., He, R., and Li, J. (2016). The more the better? A meta-analysis on effects of combined cognitive and physical intervention on cognition in healthy older adults. Ageing Res. Rev. 31, 67-79. doi: 10.1016/j.arr. 2016.07.003

Åhman, H. B., Giedraitis, V., Cedervall, Y., Lennhed, B., Berglund, L., McKee, K., et al. (2019). Dual-task performance and neurodegeneration: correlations between timed up-and-go dual-task test outcomes and alzheimer's disease cerebrospinal fluid biomarkers. J. Alzheimers Dis. 71, S75-S83. doi: 10.3233/ JAD- 181265

Conflict of Interest: The authors declare that the research was conducted in the absence of any commercial or financial relationships that could be construed as a potential conflict of interest.

Copyright (c) 2021 Jardim, Bento-Torres, Costa, Carvalho, Pontes, Tomás, Sosthenes, Erickson, Bento-Torres and Diniz. This is an open-access article distributed under the terms of the Creative Commons Attribution License (CC BY). The use, distribution or reproduction in other forums is permitted, provided the original author(s) and the copyright owner(s) are credited and that the original publication in this journal is cited, in accordance with accepted academic practice. No use, distribution or reproduction is permitted which does not comply with these terms. 